\title{
Transport of nonsorbing solutes in a streambed with periodic bedforms
}

Guangqiu Jin ${ }^{1, \#}$, Hongwu Tang ${ }^{1}$, Badin Gibbes ${ }^{2}$, Ling $\mathrm{Li}^{2,3}$, D. A. Barry ${ }^{4}$

${ }^{1}$ Centre for Eco-Environment Modelling, Hohai University, Nanjing, China

Emails: jingq@ hhu.edu.cn,

hwtang@hhu.edu.cn

${ }^{2}$ School of Civil Engineering, The University of Queensland, Queensland, Australia

Emails: b.gibbes@uq.edu.au,

1.li@uq.edu.au

${ }^{3}$ National Centre for Groundwater Research and Training, The University of

Queensland, Queensland, Australia

${ }^{4}$ Laboratoire de technologie écologique, Institut d'ingénierie de l'environnement, Ecole polytechnique fédérale de Lausanne, Station 2, CH-1015, Lausanne, Switzerland Email: andrew.barry@epfl.ch

Journal: Advances in Water Resources

${ }^{\#}$ Author to whom all correspondence should be addressed. Tel: +86 (25) 8378-6919. Fax: +86 (25) 8378-6919 


\section{Abstract}

Previous studies of hyporheic zone focused largely on the net mass transfer of solutes between stream and streambed. Solute transport processes within the bed has attracted less investigation. In this study, we combined flume experiments and numerical simulations to examine solute transport processes in a streambed with periodic bedforms. Solute originating from the stream was subjected to advective transport driven by pore water circulation due to current-bedform interactions as well as hydrodynamic dispersion in the porous bed. The experimental and numerical results showed that advection played a dominant role at the early stage of solute transport, which took place in the hyporheic zone. Downward solute transfer to the deep ambient flow zone was controlled by transverse dispersion at the later stage when the elapsed time exceeded the advective transport characteristic time $t_{c}\left(=L / u_{c}\right.$ with $L$ being the bedform length and $u_{c}$ the characteristic pore water velocity). The advection-based pumping exchange model was found to predict reasonably well solute transfer between the overlying water and streambed at the early stage but its performance deteriorated at the later stage. With dispersion neglected, the pumping exchange model underestimated the long-term rate and total mass of solute transfer from the overlying water to the bed. Therefore both advective and dispersive transport components are essential for quantification of hyporheic exchange processes.

Key words: Solute transport; Bedform; Advection; Dispersion; Hyporheic zone; Mass flux 


\section{Introduction}

The hyporheic zone (HZ, Fig. 1a) is broadly defined as a saturated transition zone between surface water and groundwater bodies [1]. This transition zone is often an important component of the riverine habitat, especially for obligate and facultative species [2-5]. As the interface between two quite different water bodies, the hyporheic zone exhibits complexities in its physical, chemical and biological characteristics, which result from intensive mixing of groundwater and surface water [6-9].

The hyporheic zone plays an important role in pollutant and nutrient transport, energy/heat transfer, and carbon cycling within a river/stream system [10-15]. It is a dynamic zone that experiences active exchange of mass (solute) and energy (heat) with the overlying stream water $[13,16]$. At the local scale, the exchange is largely affected by stream water flows, stream network morphology and bedforms, and sediment properties [17-23]. Solute exchange between the hyporheic zone and the stream affects significantly the stream water quality and ecology. For example, eggs of salmonids deposited in the gravel bed of a stream rely on dissolved oxygen (DO) transferred from the overlying water to survive and hatch $[3,24]$. On the other hand, if dissolved organic carbon is also transferred into the gravel bed and competes for DO, the pore water quality in the bed can deteriorate, affecting the salmonids' reproduction [25]. An understanding of the mechanisms that drive hyporheic exchange is required in order to better investigate the transport and fate of environmentally important substances such as nutrients and toxic contaminants in streams and rivers.

A key mechanism of hyporheic exchange is provided by hyporheic flows that are 
induced by streambed roughness features, such as bedforms. Through laboratory flume experiments, researchers have previously demonstrated an important transport pathway of solute entering the streambed on a bedform's stoss face, following a semi-circular flow path and exiting to the water column on the lee face (Fig. 1; [7, 17, 23]. Cardenas and Wilson [19] as well as Salehin [26] elucidated the pore water flow processes in a bed with triangular bedforms using numerical simulations. They showed that current-bedform interactions induced hydraulic head gradients across the sediment-water interface, which in turn generated pore water circulation in the streambed (Fig. 1b). Their simulations also showed an underflow in the deeper part of the streambed. While the underflow was induced by the hydraulic head difference between the lateral boundaries of the simulation domain (Fig. 1c), it might be thought to reflect the influence of an ambient shallow groundwater flow that often exists underneath the streambed. In reality, the shallow groundwater may even discharge to the river, creating an ambient flow condition different to that of underflow $[18,27]$. Thus the streambed may be divided into two different parts: a hyporheic zone in direct exchange with the overlying stream (interfacial exchange zone) and an ambient flow zone affected by regional groundwater conditions (Fig. 1c) [19, 28]. The hyporheic zone can also be affected by larger scale flows induced by river pools and riffles, bars and bends, and regional hydraulic gradient in the shallow aquifer [26, 29-35].

Two methods have been used to represent and quantify the exchange between a streambed and the overlying water in previous studies. With the first method, the exchange is treated as a diffusive [36-39] or a first-order mass transfer process [8, 40]. 
In most applications, the exchange parameters involved in this method were determined empirically by fitting model predictions to exchange rate data obtained either from field observations or laboratory experiments. Zaramella et al. [41] showed that the transient storage model (TSM) based on the mass transfer approximation was able to replicate advective exchange between a river and a shallow bed (with the exchange layer restricted by the presence of an impermeable boundary). However, the TSM did not predict well the riverine exchange with a relatively deep sediment bed, where flow along different advective paths in the bed yielded a wide distribution of exchange times. With the second method, the exchange model is based on direct consideration of advective transport processes with dispersion neglected [42]. Flows into and out of the streambed are controlled by the hydraulic head variation over bedforms at the sediment-water interface. The solute fluxes into and out of the bed are related to the solute residence time in the bed and calculated within stream tubes. A transfer function can then be used to determine the net mass transfer across the bed surface.

Combined effects of advection and dispersion on hyporheic exchange have been examined previously. Dispersion was introduced to the advective model and incorporated into the calculation of solute residence time within stream tubes through a random walk procedure [16, 43-45]. The dispersive effect, however, was found to be negligible for quantifying the mass exchange across the streambed with bedforms $[16$, 26, 42, 46-50]. Direct simulations of advective and dispersive transport in the streambed have also been carried out [51]. These simulations focused on solute residence time distributions and have not yet been validated against experimental data. 
Many experiments and numerical simulations have been conducted to examine the effects of bedforms on the hyporheic exchange. These studies largely concentrated on the net mass transfer of solutes, metals and/or colloids between the stream and the bed $[14,23,42,46-50,52,53]$. In contrast, solute transport in the streambed has not been investigated extensively (Fig. 1d). Although some data exist on the solute concentration profile over depth $[23,46]$, their poor spatial resolution limits their value for detailed study of solute transport processes. For example, the depth profiles of solute concentration at both the stoss and lee slope have not been measured simultaneously. Yet these profiles are thought to be different and important for analyzing the solute transport behavior at the entry (stoss) into and exit (lee) from the bed. Elliott and Brooks [23] tracked the front of the solute transport using a tracer in a laboratory flume experiment. The tracer concentration in the vicinity of the front was not reported thus limiting the use of the data for quantitative analysis. In terms of methods for quantifying hyporheic exchange, validation of the two different approaches based on dispersive and advective models respectively requires more detailed data for testing the assumptions made in each model, in particular, the neglect of dispersion in the second approach.

To improve our understanding of bedform-induced hyporheic exchange and underlying processes, we conducted laboratory flume experiments that aimed to explore the hydrodynamic interactions of a unidirectional stream flow above bedforms with pore water flow and solute transport in the underlying sediment. Thibodeaux and Boyle [17] as well as Cardenas and Wilson [19] illustrated, using experiments and numerical 
simulations, solute transport pathways in the streambed from the stoss slope to the lee slope of the bedform. To examine the solute transport behavior along such pathways, we collected data from two vertical arrays of sampling ports, located at the stoss and lee slopes of a bedform. Two-dimensional simulations of coupled flow and solute transport were conducted in parallel to the experimental investigation. Through the experiments and numerical simulations, we addressed the following questions: (1) What is the dominant mechanism underlying hyporheic exchange in a stream bed with bedforms? and (2) What are the effects of hydrodynamic dispersion on solute transport in the streambed? The results also contribute to growing experimental data on hyporheic exchange processes and solute transport in the streambed.

\section{Laboratory experiments}

\subsection{Flume setup}

A recirculating, tilting flume consisting of an $11.5-\mathrm{m}$ long, $30-\mathrm{cm}$ wide, $50-\mathrm{cm}$ deep rectangular channel (Fig. 2) was used in the experiments. Figure 2 shows ancillary experimental equipment. Surface water flow was controlled by a valve and measured with an electromagnetic flow meter.

To reduce flow turbulence generated in the pipes and the diverging section, two energy dissipation devices were installed: a perforated PVC cylinder (50-cm height and 16-cm diameter) and a perforated PVC flat in the inlet section. A curved, sealed box (70-cm length and a maximum height of $10 \mathrm{~cm}$ ) was placed in the inlet area to initiate a sand layer (streambed) with bedforms similar to those used in previous flume 
experiments $[23,46]$. At the downstream end, a water-pervious, perforated Perspex sheet covered by a fine mesh was used to hold the sand in place. In order to keep the same overlying water depth on all bedforms, we installed an adjustable-height discharge gate in the outlet section.

Two vertical arrays of sampling ports, with 1-cm intervals between neighboring ports, were installed through the sides of the flume for pore water sampling at the stoss and lee slopes of a bedform.

\subsection{Bed sand properties and preparation}

A natural river sand from the Yangtze River, with $d_{50}=0.30 \mathrm{~mm}$, was used. The bulk porosity was measured using the water evaporation method [54] as 0.33 and the saturated hydraulic conductivity, measured using the constant-head method [54], was found to be $8.84 \times 10^{-4} \mathrm{~m} \mathrm{~s}^{-1}$.

Prior to the experiment, the sand was washed (prior to transfer to the experimental flume) following a five-step procedure: (1) Washed four times with deionized water to remove impurities. Each washing cycle took approximately $45 \mathrm{~min}$. (2) Washed in a hydrochloric acid solution $(\mathrm{pH}=3.5)$ for approximately $12 \mathrm{~h}$ to remove adsorptive metals on sand particles. (3) Washed twice again using deionized water for 45 min each time. (4) Washed in a solution $(\mathrm{pH}=10.5)$ of sodium hydroxide for approximately $12 \mathrm{~h}$ to remove clays, dust and organic coatings. (5) Finally washed four times with deionized water (45 minutes each time). The sand was then stored in a bin with deionized water. 
After each experiment the sand was removed from the flume and rinsed thoroughly with deionized water in a sand-washing apparatus before the next experiment, following the same procedure as used in previous experimental designs [23, 46]. This procedure effectively removed all $\mathrm{NaCl}$ from the sand and residual pore water.

\subsection{Experimental conditions and measurements}

The experiments aimed to examine solute transport processes in the streambed with stationary bedforms. A key challenge was to create regular and uniform triangular bedforms that would remain stationary (i.e., no migration, erosion or sediment transport). In previous experiments, artificial stationary bedforms were formed under either dry or wet conditions $[16,52]$. With the dry method, the flume is filled with dry sand to a predetermined height and the bedforms are then built using a template. Afterwards the flume is filled with the deionized water. However, the last step tends to disturb the bedforms and often leaves considerable residual air in the sand, thus affecting hydraulic conductivity of the porous medium. To avoid these complications, we adopted the wet method by filling the flume with water and then sand. In making the bedform, we improved the previous method by using a perforated template with to avoid strong local flows, which would be otherwise generated and affect the bedform formation $[23,46]$. The next step was to carefully establish the desired flow condition whilst avoiding bedform disturbance. Both the flow-control valve and the height of the discharge gate were adjusted as the pump was opened slowly. It was also important to rectify the flume's slope to keep the same overlying water depth on all bedforms. The 
bedforms were found to remain stationary when the overlying water velocity was less than $17 \mathrm{~cm} \mathrm{~s}^{-1}$ in the experiments.

Lissamine, Amino $\mathrm{G} \mathrm{Acid,} \mathrm{LiCl}$ and $\mathrm{NaCl}$ have been used as conservative tracers in previous experiments $[23,46,48,52,55]$. In this study, we chose $\mathrm{NaCl}$ for the convenience of its measurement through electrical conductivity (EC). The relationship between concentration and $\mathrm{EC}$ of the $\mathrm{NaCl}$ solute at low concentrations is linear. It should be noted that the salt concentration increases the solute density, and spatial variations in concentration can lead to solute density gradients that are capable of driving flows in the sediments. Boano et al. [56] demonstrated that such density-driven flows contribute significantly to hyporheic exchange across a flat streambed made of sand. However, for a streambed with bedforms, flows driven by hydraulic gradients due to current-bedform interactions are likely to dominate the exchange process [26, 47-50].

A self-logging water quality meter (using MPTROLL 9500 manufactured by In-situ Incorporation) was used to measure electrical conductivity (EC), temperature and $\mathrm{pH}$ of the stream water at the end of the flume every $3 \mathrm{~min}$. At the beginning of the experiment, a measured quantity of $\mathrm{NaCl}$ solution was added to the reservoir after the flume had run for $20 \mathrm{~min}$ (allowing the surface water flow to reach the steady state). To quickly achieve a uniform concentration (e.g., $2.22 \mathrm{~g} \mathrm{l}^{-1}$ for Run 1; Table 1) in the surface water, the solute was added over the period of time it took for water to circulate around the system. To monitor solute transport processes in the streambed, pore water samples were taken over time from the two arrays of sampling ports. A 100- $\mu$ l syringe with a fine needle was used for sampling. Samples were taken through silica rubber 
septa in the flume walls [16]. A volume of $100 \mu \mathrm{l}$ of pore water was extracted for each sample. The relatively small sampling volume was unlikely to affect significantly the flow field in the vicinity of the sampling port [16]. The time interval of pore water sampling was $25 \mathrm{~min}$ at the beginning of the experiment to capture the relatively rapid concentration change as the solute was transferred into the bed. As the experiment continued, the concentration change became gradual after the passage of the local solute front. The sampling time interval was increased to hours towards to the end of the experiment. The water sample was diluted with $5 \mathrm{ml}$ of deionized water to allow sufficient volume for the subsequent EC measurement (using DDSJ-308A manufactured by Shanghai Precision and Scientific Instrument Company). As indicated above, to avoid disturbance on flow and solute transport in the bed, only one sample of a small volume was extracted at each sampling point and time. Due to this constraint, duplicate or triplicate samples for direct assessment of possible errors with EC measurements were not taken. However, a large number of experiments (two shown in the paper) were conducted and showed consistent behavior of tracer movement in the bed, giving confidence on the data.

Flume experiments were conducted with a range of experimental conditions. The sediment bed in the flume was 10-m long with depths varying between 12 and $15 \mathrm{~cm}$, bedform height between 1.5 and $2.5 \mathrm{~cm}$, and bedform lengths of between 15 and $20 \mathrm{~cm}$. The depth of overlying water was between 6 and $10 \mathrm{~cm}$, and the stream velocity ranged from 8 to $13 \mathrm{~cm} \mathrm{~s}^{-1}$. The temperature of the flume system was maintained at $20^{\circ} \mathrm{C}$. Each experiment was completed when the $\mathrm{NaCl}$ concentration in the overlying stream water, 
as monitored by the self-logging water quality meter, reached a relatively constant level. The results from these experiments are similar as shown in Fig. 4 and thus we focused on a typical set of results (Run 1 given in Table 1).

\section{Numerical simulations}

A one-way sequential coupling method was used to simulate the stream water flow, and pore water flow and solute transport in the streambed. First, the stream water flow was simulated and the associated pressure distribution at the sediment-water interface inferred. Then, the pore water flow in the bed, driven by the interfacial pressure variations was simulated. Finally, solute transport was simulated based on the pore water flow field (Fig. 3). This method largely follows that of Cardenas [13].

\subsection{Mathematical model and boundary conditions for overlying water}

The FLUENT finite-volume based software [53] was used to simulate numerically steady-state, two-dimensional turbulent flow over the bedforms. The governing equations for the simulation were the continuity equation and Reynolds-Averaged Navier-Stokes (RANS) equations for an incompressible, homogeneous fluid. These equations, written in the Cartesian tensor notation, are as follows (e.g., [26]):

$$
\begin{gathered}
\frac{\partial U_{i}}{\partial x_{i}}=0 \\
\rho U_{j} \frac{\partial U_{i}}{\partial x_{j}}=-\frac{\partial P}{\partial x_{i}}+\frac{\partial}{\partial x_{j}}\left(\mu \frac{\partial U_{i}}{\partial x_{j}}-\rho \overline{u_{j}^{\prime} u_{i}^{\prime}}\right)
\end{gathered}
$$

where $\rho$ and $\mu$ are water density and dynamic viscosity, respectively; $U_{i}(i=1,2)$ 
is the time-averaged velocity in the $x_{i}$ direction $(i=1,2$ for the horizontal direction and vertical directions, respectively, Fig. 1d); $P$ is time-averaged pressure; and $\overline{-u_{j}^{\prime} u_{i}^{\prime}}$ is the Reynolds stress tensor. Note that the gravitational potential is implicit in the pressure term of the above equation since the gravity is not expressed explicitly for a fixed boundary problem as simulated here (with the free surface approximated by a rigid, slip boundary) [15]. The Reynolds stresses are related to the mean rates of strain by the eddy-viscosity relation, i.e.,

$$
\overline{-u_{j}^{\prime} u_{i}^{\prime}}=v_{t}\left(\frac{\partial U_{i}}{\partial x_{j}}+\frac{\partial U_{j}}{\partial x_{i}}\right)-\frac{2}{3} k \delta_{i j}
$$

where $v_{t}$ is the kinematic eddy viscosity, $k$ is turbulent kinetic energy, and $\delta_{i j}$ is the Kronecker delta. We adopted the $k$ - $\omega$ turbulence closure scheme [57] since it has been demonstrated to work well for separated flows with adverse pressure gradients, including flow over dunes where pronounced eddies exist $[26,28,58]$. The eddy viscosity in this closure scheme is given by:

$$
v_{t}=k / \omega
$$

where $\omega$ is the ratio of the turbulence dissipation rate $\varepsilon$ to $k$ :

$$
\omega=\frac{\varepsilon}{\beta^{*} k}
$$

and $\beta^{*}$ is a closure coefficient. The steady-state transport equations for $k$ and $\varepsilon$ are:

$$
\begin{gathered}
\left.\frac{\partial\left(U_{j} k\right)}{\partial x_{j}}=-\frac{\partial}{\partial x_{j}}\left[\left(\frac{v_{t}}{\sigma_{k}}+v\right)\right) \frac{\partial k}{\partial x_{i}}\right]-\overline{u_{j}^{\prime} u_{i}} \frac{\partial U_{i}}{\partial x_{j}}-\beta^{*} k \omega \\
\left.\frac{\partial\left(U_{j} \omega\right)}{\partial x_{j}}=-\frac{\partial}{\partial x_{j}}\left[\left(\frac{v_{t}}{\sigma_{\omega}}+v\right)\right) \frac{\partial \omega}{\partial x_{i}}\right]-\alpha \frac{\omega}{\kappa}\left(\overline{-\overline{u_{j} u_{i}^{\prime}}} \frac{\partial U_{i}}{\partial x_{j}}\right)-\beta \omega^{2}
\end{gathered}
$$

where $v$ is the kinematic viscosity of water, and the values of the various model 
constants are $\alpha=5 / 9, \beta=3 / 40, \beta^{*}=9 / 100$ and $\sigma_{k}=\sigma_{\omega}=1 / 2$ [57].

The free surface was approximated as a frictionless and rigid lid, by specifying zero shear stresses at the fixed surface elevation (Fig. 3a). It is treated as a symmetric boundary in the FLUENT simulation package. Such an approximation of the free surface has been commonly used and demonstrated to be sound for conditions with small bedforms relative to the overlying water depth; and it enables efficient simulations with a fixed model domain $[26,28,58,59]$.

The horizontal extent of the model domain included only one bedform. We imposed spatially periodic pressure and velocity boundary conditions on the two vertical sides of the surface water domain (and the porous bed). A constant flux condition was specified in the simulation of the surface water flow. This resulted in a pressure drop $(\mathrm{d} P)$, i.e., $P\left(0, x_{2}\right)=P\left(L, x_{2}\right)+\mathrm{d} P$, between the two sides (Fig. 3a). Pressure was also specified at the upper right or lower right corner of the RANS domain in order to achieve a unique solution [19].

The sediment-water interface was treated as a no-slip wall boundary. FLUENT uses an enhanced wall treatment for the near-wall region. The treatment is based on a near-wall modeling method that combines a two-layer model (including a laminar sub-layer and a fully-turbulent outer region) with enhanced wall functions [60].

To represent accurately the geometry of the domain, we used AutoCAD to draw the bedforms and then exported the data file to FLUENT. The simulation domain was then discretized by structured grids with quadrilateral mesh elements. The grid was refined near the sediment-water interface. There were 50,000 - 160,000 elements 
within a single bedform in the simulations with grid sizes ranging between $0.4 \mathrm{~mm}$ and $9 \mathrm{~mm}$. FLUENT uses a time-marching scheme to solve the steady flow problem. The iteration proceeds until a steady-state solution is reached with the maximum relative difference between computed flow variables $(u, v, p, k, \omega)$ of two consecutive iteration steps dropped below $10^{-5}$.

\subsection{Mathematical model and boundary conditions for pore water}

COMSOL Multiphysics, a finite element-based software [54], was used to numerically simulate flow and solute transport in the bed. The pore water flow is governed by the combination of Darcy's Law and the continuity equation for incompressible flow in a non-deformable medium; hence the groundwater flow equations are:

$$
\begin{gathered}
\frac{\partial u_{i}}{\partial x_{i}}=0 \\
u_{i}=-\frac{K}{\theta} \frac{\partial h}{\partial x_{i}}
\end{gathered}
$$

where $\theta$ is the bulk porosity of the sand bed; $K$ is the hydraulic conductivity of the bed, $=\kappa g / v$ ( $\kappa$ is the intrinsic permeability of sand and $g$ is the magnitude of gravitational acceleration); $u_{i}(i=1,2)$ is pore water flow velocity component in the $x_{i} \quad(i=1,2)$ direction; and $h$ is hydraulic head, $=p / \rho g+x_{2}$ with $p$ being the pore water pressure. Note that the above governing equations of the pore water flow are based on a constant fluid density. In other words, the effect of density variations on the flow is assumed to be small compared with that of hydraulic head gradients on the sediment-water interface generated by current-bedform interactions. 
The overlying water flow model provides the pressure distribution at the sediment-water interface. Note that the gravity force is inherent in the pressure term in the surface water flow equation (2) and hence computed pressure values $(P)$ represent the hydraulic heads $(h)$ at the sediment-water interface. This pressure solution is thus imposed as a Dirichlet boundary condition at the top boundary of the streambed domain (Fig. 3a; [26, 28]). The lateral (vertical) boundaries were also set as periodic velocity and pressure boundaries with an imposed pressure/head drop, i.e., $u_{i}\left(0, x_{2}\right)=u_{i}\left(L, x_{2}\right)$ and $h\left(0, x_{2}\right)=h\left(L, x_{2}\right)+\mathrm{d} P / \rho g$ (where $\mathrm{d} P$ is the pressure drop between the two side boundaries of the surface water domain). The pressure drop results in what is commonly referred to as "underflow" [28]. As discussed in the introduction, in reality such underflow may exist due to regional hydraulic gradients in the shallow groundwater aquifer below the hyporheic zone. The bottom boundary was set as a no flow boundary (Fig. 3a).

The $\mathrm{NaCl}$ solute transport in the pore water is modeled based on the $2 \mathrm{D}$ mass transport equation (for a nonreactive solute) written as [61]

$$
\frac{\partial C}{\partial t}=\frac{\partial}{\partial x_{i}}\left(D_{i j} \frac{\partial C}{\partial x_{j}}-u_{i} C\right)
$$

where $C$ is the solute concentration, $t$ is time, $u_{i}(i=1,2)$ is pore water flow velocity component in the $x_{i}(i=1,2)$ direction, and $D_{i j}$ is the component of the 2D dispersion coefficient tensor:

$$
\begin{gathered}
D_{i i}=\alpha_{L} \frac{u_{i}^{2}}{|u|}+\alpha_{T} \frac{u_{j}^{2}}{|u|}+D_{e} \\
D_{i j}=D_{j i}=\left(\alpha_{L}-\alpha_{T}\right) \frac{u_{i} u_{j}}{|u|}+D_{e}
\end{gathered}
$$


Here, $\alpha_{L}$ is the longitudinal dispersivity, a property of the porous medium describing dispersive transport in the flow direction; and $\alpha_{T}$ is the transverse dispersivity, describing dispersive transport normal to the flow direction. The absolute value $|u|=\sqrt{u_{1}^{2}+u_{2}^{2}}$ is the magnitude of the pore water flow velocity. $D_{e}$ is the effective molecular diffusion coefficient in the porous medium.

The boundary conditions for the solute transport are set as follows (Fig. 3b): (1) on the lateral boundaries $\left(x_{1}=0\right.$, and $\left.x_{1}=L\right)$ of the domain, the periodic conditions $C\left(0, x_{2}, t\right)=C\left(L, x_{2}, t\right)$ and $\partial C\left(0, x_{2}, t\right) / \partial x_{2}=\partial C\left(L, x_{2}, t\right) / \partial x_{2}$ were imposed; (2) on the bottom of the domain, a no-flux condition applies, giving zero concentration gradient $\partial C /\left.\partial x_{2}\right|_{x_{2}=0}=0$ since local vertical flow and hence advective flux is zero at this boundary; (3) along the sediment-water interface, the boundary condition was set as follows:

$$
\begin{cases}C=C_{t} & \mathbf{n} \bullet \mathbf{u} \geq 0 \\ \frac{\partial C}{\partial \mathbf{n}}=0 & \mathbf{n} \bullet \mathbf{u}<0\end{cases}
$$

where $\mathbf{n}$ is the unit vector normal to the interface (pointing inward), $\mathbf{u}$ is the pore water flow velocity vector, and $C_{t}$ is the concentration of solute in the overlying water. It should be noted that the overlying water is relatively well mixed as shown in our experiments and hence a uniform concentration was assumed there. To simulate the flume experiments, $C_{t}$, varying with time as a result of the mass exchange between the overlying water and the bed, was calculated as follows:

$$
C_{t}=\frac{C_{0} V_{s}-B \iint_{A} \theta C\left(x_{1}, x_{2}, t\right) d A}{V_{s}}
$$

where $C_{0}$ is the initial solute concentration of the overlying water, $V_{s}$ is the total 
volume of water in the flume system excluding pore water in the streambed, $C\left(x_{1}, x_{2}, t\right)$ is the simulated solute concentration of pore water at location $\left(x_{1}, x_{2}\right)$ at time $t, B$ is the width of the flume, and $A$ is the area (on the $x_{1}-x_{2}$ plane) of the sand bed in the flume. The integral term in equation (14) represents the amount of $\mathrm{NaCl}$ stored in the bed.

Additional numerical simulations were conducted to examine dispersion effects (presented in Section 5.2). For the purpose of simplicity, the solute concentration in the overlying water in these simulations was kept constant over the simulation period, i.e., $C_{t}=C_{0}$

\section{Experimental and numerical modeling results}

The flume is a closed system in our experiments. As discussed above, the quantity and concentration of solute in the overlying water decrease as the solute is transferred into the bed. This trend has been featured in the model predictions based on equation (14). To better match the experimental data, we adjusted the hydraulic conductivity of the sand from the measured value $8.84 \times 10^{-4} \mathrm{~m} \mathrm{~s}^{-1}$ to $1.2 \times 10^{-3} \mathrm{~m} \mathrm{~s}^{-1}$ and the bulk porosity from the measured value 0.33 to 0.39 . Although the adjustment was based on the fitting of model predictions to data, such small variations in $K$ and $\theta$ would not be unexpected since the sand packing method in the flume experiments was different from that used in measuring both parameters. While sand was added to the flume when it was full of water, the permeameter was filled with sand to a height of $5 \mathrm{~cm}$ and then filled slowly with water from the bottom to the top of the sand each time until the height of 
the sand column reached $30 \mathrm{~cm}$. The longitudinal dispersivity $\left(\alpha_{L}\right)$ of the bed was set to $1 \mathrm{~mm}$ and the ratio of transverse dispersivity to longitudinal dispersivity $\left(\alpha_{T} / \alpha_{L}\right)$ was assumed to be a constant, $1 / 10$ [39, 62-64]. To determine the effective molecular diffusion coefficient, we used the following formula $[65,66]$,

$$
D_{e}=\frac{D_{w}}{\theta^{(1-m)}}
$$

where $D_{w}$ is the free solution diffusion coefficient in water $\left(1.61 \times 10^{-9} \mathrm{~m}^{2} \mathrm{~s}^{-1}\right.$ at $25^{\circ} \mathrm{C}$ for sodium chloride in water), $\theta$ is the porosity, and $m$ equals 2 for $\theta \leq 0.7$ and 3 for $\theta>0.7$. This gives an effective molecular diffusion coefficient of $5 \times 10^{-10} \mathrm{~m}^{2} \mathrm{~s}^{-1}$ for the sand bed.

The results from both the experiments and numerical simulations are described in the following sections. The flow simulation results were essential for predictions of solute transport and concentration variations in both the stream and bed. Although no pore water flow measurements were made to validate the simulation results due to the constraints of the apparatus, the performance of the flow model is indicated in the comparison between the predicted and measured concentration profiles presented below.

\subsection{Temporal variation of solute concentration in the overlying water}

The solute concentration in the overlying water experienced a sharp decrease at the beginning of the flume experiment, followed by a gradual decline phase (squares in Fig. 4). The sharp decrease corresponds to the initial rapid transfer of solute from the overlying water to the shallow streambed area where the pore water flow velocity and hence solute transport flux were relatively large. After the solute front passed through 
this shallow bed area, the solute concentration in the overlying water continued to decrease gradually as the solute was transferred slowly to the deep streambed area. In the figure, we also plot the simulation results based on measured and adjusted hydraulic conductivity $K$ and bulk porosity $\theta$ for comparison. Overall the model predicted the trend observed in the experiment. With the adjusted $K$ and $\theta$ values, the model predictions matched the data well (Fig. 4). The measured $K$ and $\theta$ values were slightly lower than the fitted values, both giving slower solute transfer to the bed and hence a more gradual decline of solute concentration in the overlying water. If the experiment and simulation continued to run for a sufficiently long time, the solute concentration in the pore water would become the same as that in the overlying water, i.e., the system reaching a steady state with no net exchange between the overlying water and pore water. The asymptotic solute concentration (dash-dotted line in Fig. 4) in both the overlying water and pore water can be determined according to $C_{0} V_{s} /\left(V_{s}+V_{p}\right) \quad\left(V_{p}\right.$ is the total volume of the pore water in the streambed). The asymptotic solute concentration calculated using measured $\theta$ was higher than that based on adjusted $\theta$ because measured $\theta$, less than adjusted $\theta$, gave a smaller $V_{p}$.

\subsection{Spatial and temporal variations of solute concentrations in the bed}

As mentioned, two vertical arrays of sampling ports were installed to monitor the variation of solute concentration in the pore water. Array N1 was located at the stoss slope of a bedform where the solute entered the streambed, and array N2 located at the lee slope of the bedform where the solute exited the streambed (Fig. 5). The distinction between these two sampling areas is thought to be important for studying the solute 
transport behavior under the influence of circulation induced by current-bedform interactions. Previous experiments had not made such a distinction particularly [23, 46]. The depth profiles of measured solute concentration at both sampling locations and various times are displayed in Fig. 6 in comparison with the model predictions. Results from numerical simulations with measured and adjusted $K$ and $\theta$ values are also compared. Overall the model simulated the observations well (with coefficients of regression between model prediction and data equal to $0.973,0.929,0.981$ for the case with measured $K$ and $\theta$, measured $K$ and adjusted $\theta$, and adjusted $K$ and $\theta$, respectively). These profiles appeared to show a solute front propagating downward at both locations. However, these trends did not simply reflect a downward solute movement but resulted from both horizontal (e.g., from N1 to N2) and vertical solute transport through pore water circulation cells (flow tubes). For example, the concentration at a deep port of N2 (N2_P9) would not correspond to the concentration in the shallow area (ports above) of this sampling location. Instead, it was related to the concentration near a port of $\mathrm{N} 1$ (N1_P10) given that both ports were along the same circulation path (Fig. 5). Such transport behavior will be further analyzed in the next section.

It is interesting to note that the concentration seemed to increase slightly with the depth to a maximum value near the solute front prior to a rapid decrease to zero. This behavior corresponds to the reduction of the solute concentration in the overlying water with time. Larger solute concentrations at deeper ports of N1 and N2 at a particular time were linked to higher solute concentrations in the overlying water at earlier times since the deeper ports were associated with longer and slower circulation paths. The 
maximum concentration would be expected to occur at the depth where a solute front initiated from the sediment-water interface just arrived at the time. It is also evident that, as time went on, the maximum concentration over the depth at both $\mathrm{N} 1$ and $\mathrm{N} 2$ decreased, occurring deeper in the array. This was partly due to the dispersion effect as analyzed in the next section.

The trends in the pore water solute concentration are further displayed in Fig. 7 where the temporal variations of solute concentration at various sampling ports are presented. Located "downstream", N2 lagged N1 in the concentration response to the solute transport processes. At both locations, the time series exhibit two phases of solute concentration changes at all sampling ports except those near the bottom of the streambed: a rapid increase phase followed by a gradual decline. The first phase was due to the initial arrival of solute that was transferred from the overlying water to the streambed. The second phase reflected adjustment of local pore water solute concentration as a result of continuing exchange between the streambed and overlying water of decreasing concentration. The local peak solute concentration, separating these two phases, corresponds to the maximum concentration shown in the depth profiles at the peak time. The peak concentration decreased with the depth, again indicating the dispersion effect that was more profound for deep ports associated with long transport paths. This behavior is essentially consistent with that in Fig. 6.

The differences among the simulation results with adjusted $K$ and $\theta$, measured $K$ and adjusted $\theta$, and measured $K$ and $\theta$ were manifested mainly as different propagation speeds of local solute fronts (Fig. 6). With only $\theta$ adjusted to a slightly 
higher value, the pore water flow velocity was reduced, resulting in a slower movement of the solute front compared with that based on the measured $K$ and $\theta$. The adjustment of both $K$ and $\theta$ overturned the change to give the fastest movement of the solute front. While the fitting of the observed front movement by the numerical model could have been based on adjustment of $K$ alone, the overall exchange rate and hence the decrease of the solute concentration in the overlying water were also affected by $\theta$ as shown in Fig. 4. The solute concentration of the overlying water in turn affected the solute concentration profile above the front. The prediction with the adjusted $K$ and $\theta$ produced the lowest concentration in the above-front area compared with the results from simulations using measured $K$ and adjusted $\theta$, and measured $K$ and $\theta$. This rather subtle effect was linked to the behavior of the solute concentration in the overlying water (Fig. 4).

\section{Discussion}

As mentioned in the introduction, the exchange between the streambed and overlying water is typically quantified either as an advective transport process or through a diffusive/dispersive approximation. The purpose of the analyses presented in this section is to determine the combined effects of advection and dispersion that co-exist in the system. In particular, we are interested in conditions under which advection or dispersion may dominate.

\subsection{Advection and pumping exchange models}

Various advective models (collectively called the pumping exchange model, PEM) 
have been developed previously for quantifying the mass exchange across the streambed with bedforms [42, 46, 47]. The essence of these models is an assumption of advection alone in transporting solute through flow tubes. A further assumption of sinusoidal pressure variations along the sediment-water interface was also made to permit analytical solutions $[42,46,47]$. While the second assumption is invalid for our experiments, we will test the first assumption of solely advection using flow fields predicted by the numerical model.

A typical flow tube simulated numerically is shown in Fig. 5. The travel time of a solute "slug" through this flow tube can be calculated based on the simulated flow velocity and used to determine the initial arrival of solute at various points along the tube. With both longitudinal and transverse dispersions neglected, the arrival of a solute "slug" would lead to a step change of local solute concentration. The concentration $\left(C_{s}\right)$ at a sampling port would then be related to the concentration at the entry of its associated flow tube (i.e., the concentration of the overlying water, $\left.C_{t}\right)$ according to $C_{s}(t)$ $=C_{t}(t-T)$ for $t>T$ where $T$ is the slug travel time to the port. We computed the concentrations at various ports based on this slug (advective) transport model. The results show that slug transport did not occur in the experiment or numerical simulation (Fig. 8a). Instead the solute concentration started to increase before the arrival time of the slug due to dispersion. However, the advective transport model seemed to provide a reasonable approximation of the temporal concentration variation at the shallow ports, indicating the dominance of advection in the transport processes within the shallow area. As the solute transport length and time increased for the deep ports, the effect of the 
dispersion on the concentration profile became significant (Fig. 8a).

We analyzed further the dispersion effect based on a 1D transport model for the flow tube and assuming a constant solute concentration at the entry (neglecting the variations of the solute concentration in the overlying water). Based on this model, half of the input concentration is expected to occur at a port at the slug arrival time (details in the appendix). Note that while longitudinal dispersion (with the dispersion coefficient assumed constant) is considered in the model, transverse dispersion across the flow tube has been neglected. Using the experimental results plotted in Fig. 7, we determined the time when half the local peak concentration was reached at the sampling ports (different depths) of N1 and N2. For each port, we also computed the travel time of solute (slug) through the flow tube between the tube's entry point and the port. The half peak-concentration time estimated based on the data was reasonably close to the travel time predicted by the model (Fig. 8b). The agreement indicates negligible transverse dispersion effects on solute transport in the shallow sand bed. However, as demonstrated in the next section, transverse dispersion plays an important role in transporting solute deeper into a deep bed where an extensive underflow zone exists.

To further examine the mode of overall solute transport in the streambed, we analyzed the results from the numerical simulation. Based on simulated flow velocities, we computed the travel paths of particles initially placed on the stoss slope of the bedform over different elapse times and analyzed how these paths related to the movement of solute in the bed as given by the predicted solute concentration field (Fig. 9). The results show that the travel paths tracked well the downward-moving solute 
front at early time (60 $\mathrm{min})$, when the solute front remained in the shallow area. However, as the solute front moved further down to the deep area over a longer time (2500 $\mathrm{min}$ ), it started to deviate from the particle's travel path. Such a deviation was relatively small in the experiments (with a bed of small thickness) compared with the results from numerical simulations based on a thicker bed (presented in the next section). Nevertheless, the trends were consistent with the finding from the above analysis on measured concentration time series: While advection-dominated solute transport occurred within the shallow area during the early period, dispersion became significant in the deep area over longer periods. Based on the bedform length $(L)$ and pore water flow characteristic velocity $\left(u_{c}\right)$, an advection characteristic time scale $\left(t_{c}\right)$ may be defined to separate these two periods:

$$
\begin{gathered}
t_{c}=L / u_{c} \\
u_{c}=\left(K H_{m}\right) /(L \theta)
\end{gathered}
$$

where $H_{m}$ is the amplitude of hydraulic head variation along the sediment-water interface induced by bedform-stream flow interactions [42]. For the experimental conditions, $t_{c}$ was estimated to be $688 \mathrm{~min}$. The location of the advective front given by this characteristic time is shown in Fig. 9.

\subsection{Dispersion effects}

To further analyze the effects of dispersion on the solute transport processes, we conducted numerical simulations with a deeper streambed (depth $=1 \mathrm{~m}$ ), where an extensive ambient underflow zone co-existed with the hyporheic zone (Fig. 10). For the purpose of focusing on the dispersion effects, the simulations were based on a constant 
solute concentration in the overlying water over the simulation period. Simulations were conducted with the longitudinal dispersivity varying from $0.001 \mathrm{~m}$ to $0.01 \mathrm{~m}$ and $0.1 \mathrm{~m}$ to examine the dispersion effects $[39,51]$. The large dispersivity value $(0.1 \mathrm{~m})$ used represents a highly dispersive condition that may occur in a gravel bed. Other model parameter values for these numerical simulations are listed in Table 2.

We first examined how the solute front was affected by dispersion (Fig. 10). It is clear that, as the dispersivity increases, the solute front becomes more smeared and the dispersivity affects the distribution of solute in the streambed. For a constant concentration boundary at the sediment-water interface, a representative location of the solute front may be defined by $C / C_{0}=0.5$ (see the appendix for details). Again the solute front was found to be well tracked by particles' travel paths in the shallow area, especially for cases with small dispersivity values. As the solute movement continued downward, the front departed from the particle's travel path. In the deep area, solute transport became controlled by (transverse) dispersion, completely decoupled from the particle's travel path, which ended at the divide between the circulation zone (hyporheic zone) and ambient underflow zone.

To better understand the solute transport processes in the streambed, we analyzed the advective $(\theta C \mathbf{u})$ and dispersive fluxes $\left(-\theta D_{i j} \nabla C\right)$ separately. Advection fluxes exhibit similar spatial distributions for $\alpha_{L}=0.001 \mathrm{~m}$ and $0.01 \mathrm{~m}$ (Fig. 11a). As $\alpha_{L}$ increased to $0.1 \mathrm{~m}$, different spatial patterns of the advective flux appeared, especially in the area near the solute front with a thickened dispersion/mixing zone. Overall the spatial patterns of advection flux largely followed those of pore water flow 
velocity in areas with significant solute concentrations. At the early time $\left(t<t_{c}\right.$; for the simulated condition, $t_{c}=2.20 \times 10^{4} \mathrm{~min}$ ), no solute was present in the deep area and hence no advection of solute occurred despite the local flow. As the downward solute transfer continued, significant advective fluxes appeared in the deep area except the vicinity of the stagnation point. Despite the hydraulic divide (flow separation) between the shallow (HZ) and deep (ambient underflow zone) areas, the solute was transferred from the shallow area to the deep area by transverse dispersion.

Dispersive fluxes were concentrated near the solute front in the hyporheic zone prior to solute reaching the hydraulic divide. A greater dispersivity led to a more extensive area of active dispersive transport with significant fluxes (Fig. 11b). As the solute transport continued downward, dispersive fluxes occurred increasingly below the divide, and were responsible for transferring solute from the hyporheic zone to the deep ambient flow area. By this stage, the direction of the dispersion fluxes became predominantly downward (i.e., transverse to the largely horizontal flow in the ambient underflow zone) as shown in Fig. 11b.

To further examine the relative importance of the two transport processes in different areas at different times, we computed the total vertical advective flux $\left(J_{a d v}\right)$ and the total vertical dispersive flux ( $J_{\text {dis }}$ ) integrated over one bedform (from $x_{1}=0$ to $L)$ at different distances from the bottom of the bed $\left(x_{2}\right)$ :

$$
\begin{gathered}
J_{a d v}\left(x_{2}\right)=\frac{1}{L} \int_{0}^{L} \theta u_{2} C d x_{1} \\
J_{d i s}\left(x_{2}\right)=\frac{1}{L} \int_{0}^{L}\left(-\theta D_{22} \frac{\partial C}{\partial x_{2}}-\theta D_{21} \frac{\partial C}{\partial x_{1}}\right) d x_{1}
\end{gathered}
$$

These two fluxes combine to give the total solute input to the sand volume below 
$x_{2}$ over the bedform since periodic boundary conditions were applied to the side boundaries and the bottom boundary permitted no flux. The total solute input, $J\left(x_{2}\right)$, would be in balance with the total solute accumulation in the volume [39]:

$$
J\left(x_{2}\right)=\frac{\mathrm{d} m\left(x_{2}\right)}{\mathrm{d} t}=\int_{-d_{b}}^{x_{2}} \int_{0}^{L} \theta \frac{\partial C}{\partial t}\left(x_{1}, x_{2}^{\prime}, t\right) d x_{1} d x_{2}^{\prime}
$$

where $d_{b}$ is the average depth of streambed (Fig. 3).

The results confirm the mass balance principle, i.e., $J_{a d v}+J_{d i s}=\mathrm{d} m\left(x_{2}\right) / \mathrm{d} t$. At early times, advection dominated the solute transport that took place in the hyporheic zone (Fig. 12), especially for cases with relatively small dispersivity values. However, hydrodynamic dispersion became significant after the solute front reached the hydraulic divide between the hyporheic zone and ambient flow zone (Fig. 12). The total dispersive flux provided the solute supply for the deep area. Such dispersion effects explain why the advective transient storage model cannot represent well exchange between a relatively deep sediment bed and the overlying stream [41].

The importance of dispersion is further demonstrated in Fig. 13, which plots the cumulative solute mass transferred from the overlying water to the bed. The normalized total amount of mass transfer across one bedform, $m^{*}$, is calculated as follows:

$$
m^{*}=\frac{\iint_{A_{b}} \theta C\left(x_{1}, x_{2}, t\right) d A}{\theta C_{0} A_{b}}
$$

where $A_{b}$ is the area of the bed area over one bedform. Again, the results show that dispersion played a relatively minor role early in the process when solute transport, dominated by advection, took place in the hyporheic zone. Advection determined the rate of exchange across the sediment-water interface. The solute accumulation rates in the three simulated cases with different dispersivities overlap and are well predicted by 
the (advective) pumping exchange model. However, the trends diverged as the solute transfer extended to the deep area of the bed, especially in the case of a thick bed. The time of this divergence corresponded to the advection characteristic time $\left(t_{c}\right)$ reasonably well. Dispersion became an important factor controlling the solute transfer. Although the total cumulative solute mass approached the same amount determined by the pore volume in the bed for all cases of different dispersivities, the accumulation rate increased with larger dispersivities. It is interesting to note that the pumping exchange model predicted a lower asymptotic value of accumulated mass, reflecting a smaller pore volume (within the HZ) for advective only solute transport assumed in the model.

\section{Conclusions}

Advection and dispersion are both important factors for solute exchange between stream and streambed. However, previous investigations often considered one factor and ignored the other. In this study, laboratory experiments and numerical simulations were conducted to investigate the processes and mechanisms of nonsorbing solute transport in a streambed with regular and uniform bedforms. In the experiments, data were collected from two vertical arrays of sampling ports located at the stoss and lee slopes of the bedform. We conducted two-dimensional simulations of coupled flow and solute transport in parallel to the experimental investigation. A particular focus of the study was on the relative importance of advection and dispersion in different areas at different stages of solute transport processes.

The experimental and numerical results showed that advection played a dominant 
role at the early stage $\left(t<t_{c}\right)$ of solute transport, which took place in the hyporheic zone. At this stage, the advection-based pumping exchange model was found to predict well the solute transfer between the overlying water and streambed. The dispersivity only affected the distribution of solute in the streambed, especially near the front. However, the performance of the pumping exchange model deteriorated at the later stage as the solute transfer extended to the deep ambient underflow area. Downward solute transfer to the deep area was controlled by transverse dispersion. With dispersion neglected, the classical pumping exchange model underestimated the rate and total mass of solute transfer from the overlying water to the bed.

Although the focus here was on solute originating from the overlying water, the findings also have implications for transfer of bed-origin solute to the overlying water. The flow system considered here, induced by stream flow-bedform interactions, is a common occurrence in natural streams. However, other flows such as those induced by pool-and-riffle, bars and bends, and even basin-scale hydro-morphological features co-exist in reality. How these flows of different scales combine to influence the exchange between the stream and bed remains an important question for future research. Density-driven flows, neglected in this study, may also contribute to the hyporheic exchange in streambeds with bedforms. The importance of the density effect on the flow relative to that of hydraulic head gradients due to bedform-current interactions requires further investigations. 


\section{Notation}

$A \quad$ area (on the $x_{1}-x_{2}$ plane) of the sand bed in the flume, $\left[\mathrm{L}^{2}\right]$

$A_{b} \quad$ area of the bed area over one bedform, $\left[\mathrm{L}^{2}\right]$

$B \quad$ width of the flume, [L]

C solute concentration, $\left[\mathrm{ML}^{-3}\right]$

$C_{0} \quad$ initial solute concentration in overlying water, $\left[\mathrm{ML}^{-3}\right]$

$C_{t} \quad$ solute concentration in overlying water at time $t,\left[\mathrm{ML}^{-3}\right]$

$d_{b} \quad$ average depth of streambed, [L]

$D_{e} \quad$ effective molecular diffusion coefficient in pore water, $\left[\mathrm{L}^{2} \mathrm{~T}^{-1}\right]$

$D_{i j} \quad$ components of the $2 \mathrm{D}$ dispersion coefficient tensor, $\left[\mathrm{L}^{2} \mathrm{~T}^{-1}\right]$

$D_{w} \quad$ free solution diffusion coefficient in water, $\left[\mathrm{L}^{2} \mathrm{~T}^{-1}\right]$

$H \quad$ average water depth in overlying water, [L]

$H_{b} \quad$ bedform height, [L]

$H_{m} \quad$ amplitude of hydraulic head variation along sediment-water interface, [L]

$J \quad$ mass flux, $\left[\mathrm{MT}^{-1} \mathrm{~L}^{-2}\right]$

$J_{a d v}\left(x_{2}\right) \quad$ average total vertical advection flux, $\left[\mathrm{MT}^{-1} \mathrm{~L}^{-2}\right]$

$J_{d i s}\left(x_{2}\right) \quad$ average total vertical dispersion flux, $\left[\mathrm{MT}^{-1} \mathrm{~L}^{-2}\right]$

$K \quad$ hydraulic conductivity for the streambed sand, $\left[\mathrm{LT}^{-1}\right]$

$k \quad$ turbulent kinetic energy, $\left[\mathrm{L}^{2} \mathrm{~T}^{-2}\right]$

$L \quad$ length of bedform, [L]

$L_{\mathrm{c}} \quad$ stoss length of bedform, [L]

$\mathbf{n}$

unit vector normal to the bed surface pointing into the bed, [L] 


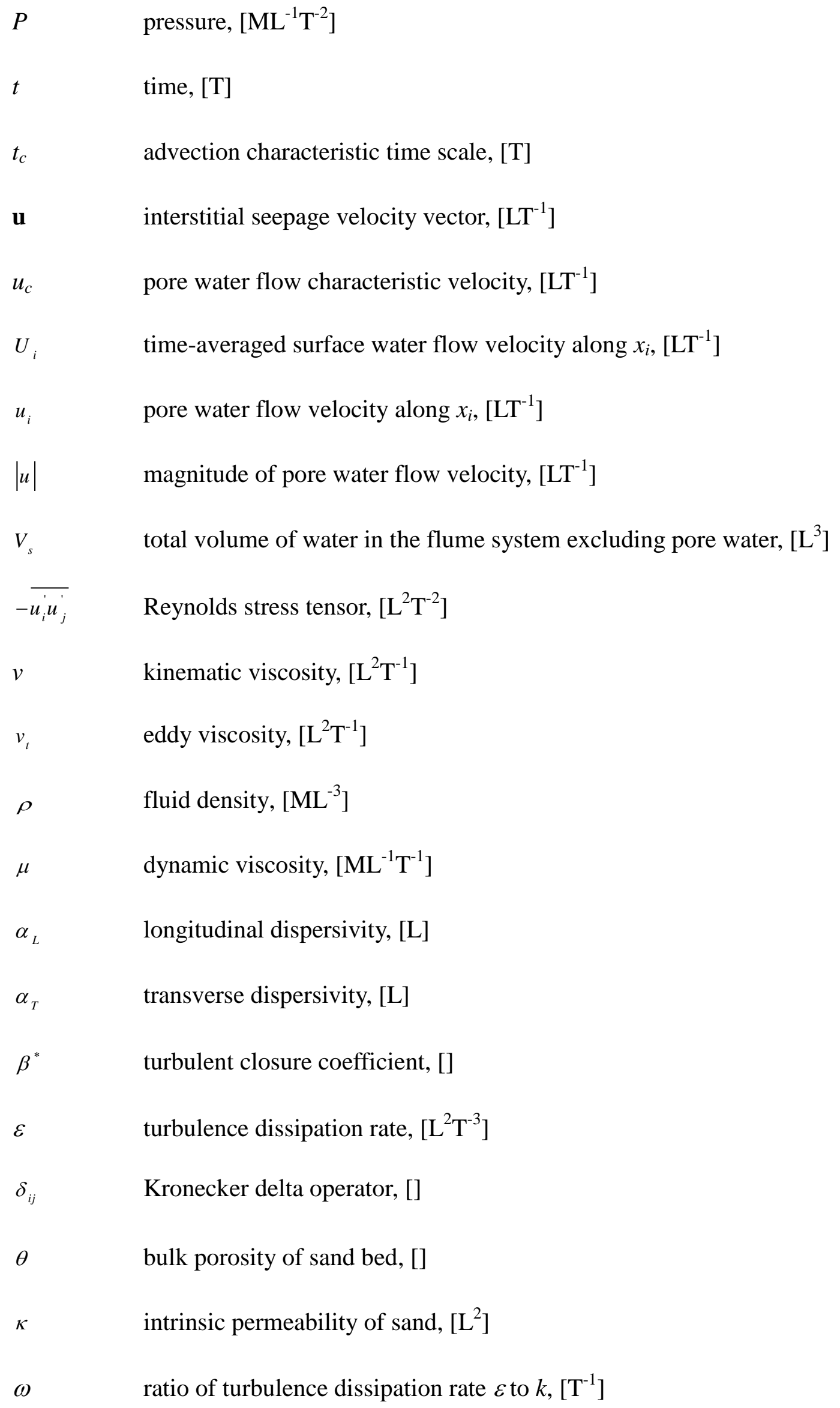




\section{Acknowledgments}

This research has been supported by the Natural Science Foundation of China (50425926), the Natural Science Foundation of Hohai University (2009422211), the Fundamental Research Funds for the Central Universities (2009B09514), and Australian Research Council (DP0988718). The authors would like to acknowledge the assistance provided by Xiaoquan Yang, Ming Chen and Kai Xie during the experiments.

\section{References}

[1] Krause S, DM Hannah, JH Fleckenstein. Hyporheic hydrology: Interactions at the groundwater-surface water interface Preface. Hydrol Process 2009;23(15):2103-7.

[2] Zimmermann AE, M Lapointe. Intergranular flow velocity through salmonid redds: Sensitivity to fines infiltration from low intensity sediment transport events. River Res Appl 2005;21(8):865-81.

[3] Malcolm IA, C Soulsby, AF Youngson, DM Hannah, IS McLaren, A Thorne. Hydrological influences on hyporheic water quality: implications for salmon egg survival. Hydrol Process 2004;18(9):1543-60.

[4] Stanford JA, JV Ward. The hyporheic habitat of river ecosystems. Nature 1988;335(6185):64-6.

[5] Baxter CV, FR Hauer. Geomorphology, hyporheic exchange, and selection of spawning habitat by bull trout (Salvelinus confluentus). Can J Fish Aquat Sci 2000;57(7):1470-81.

[6] Huettel M, H Roy, E Precht, S Ehrenhauss. Hydrodynamical impact on biogeochemical processes in aquatic sediments. Hydrobiologia 2003;494(1-3):231-6.

[7] Huettel M, W Ziebis, S Forster. Flow-induced uptake of particulate matter in permeable sediments. Limnol Oceanogr 1996;41(2):309-22.

[8] Bencala KE. Interactions of solutes and streambed sediment 2. A dynamic analysis of coupled hydrologic and chemical processes that determine solute transport. Water Resour Res 1984;20(12):1804-14.

[9] Precht E, U Franke, L Polerecky, M Huettel. Oxygen dynamics in permeable sediments with wave-driven pore water exchange. Limnol Oceanogr 2004;49(3):693-705.

[10] Boulton AJ, S Findlay, P Marmonier, EH Stanley, HM Valett. The functional significance of the hyporheic zone in streams and rivers. Annu Rev Ecol Syst 1998;2959-81.

[11] Hill AR, CF Labadia, K Sanmugadas. Hyporheic zone hydrology and nitrogen dynamics in relation to the streambed topography of a N-rich stream. Biogeochemistry 1998;42(3):285-310.

[12] Pretty JL, AG Hildrew, M Trimmer. Nutrient dynamics in relation to surface-subsurface hydrological exchange in a groundwater fed chalk stream. J Hydrol 2006;330(1-2):84-100.

[13] Cardenas MB. Dynamics of fluids, heat and solutes along sediment-water interfaces: a multiphysics 
modeling study: Ph. D. thesis, The New Mexico Institute of Mining and Technology, USA; 2006.

[14] Packman AI, NH Brooks. Hyporheic exchange of solutes and colloids with moving bed forms. Water Resour Res 2001;37(10):2591-605.

[15] Cardenas MB, JL Wilson. Effects of current-bed form induced fluid flow on the thermal regime of sediments. Water Resour Res 2007;43(8). doi:10.1029/2006WR005343.

[16] Elliott. Transfer of solutes into and out of streambeds. Rep KH-R-52, WM Keck Lab of Hydraul and Water Resour, Calif Inst of Technol, Pasadena, USA; 1990.

[17] Thibodeaux LJ, JD Boyle. Bedform-generated convective transport in bottom sediment. Nature 1987;325(6102):341-3.

[18] Cardenas MB, JL Wilson. The influence of ambient groundwater discharge on exchange zones induced by current-bedform interactions. J Hydrol 2006;331(1-2):103-9.

[19] Cardenas MB, JL Wilson. Hydrodynamics of coupled flow above and below a sediment-water interface with triangular bedforms. Adv Water Resour 2007;30(3):301-13.

[20] Packman AI, A Marion, M Zaramella, C Chen, JF Gaillard, DT Keane. Development of layered sediment structure and its effects on pore water transport and hyporheic exchange. Water, Air, Soil Pollut 2006;6(5):69-78.

[21] Packman AI, D Jerolmack. The role of physicochemical processes in controlling sediment transport and deposition in turbidity currents. Mar Geol 2004;204(1-2):1-9.

[22] Salehin M, AI Packman, M Paradis. Hyporheic exchange with heterogeneous streambeds: Laboratory experiments and modeling. Water Resour Res 2004;40(11). doi:10.1029/2003WR002567.

[23] Elliott AH, NH Brooks. Transfer of nonsorbing solutes to a streambed with bed forms: Laboratory experiments. Water Resour Res 1997;33(1):137-51.

[24] Meyer EI, O Niepagenkemper, F Molls, B Spanhoff. An experimental assessment of the effectiveness of gravel cleaning operations in improving hyporheic water quality in potential salmonid spawning areas. River Res Appl 2008;24(2):119-31.

[25] Hill AR. Stream chemistry and riparian zones. in: JB Jones, PJ Mulholland, (Eds.). Streams and ground waters. Academic, San Diego, Calif, USA; 2000. pp. 83-110.

[26] Salehin M. Hydrodynamics of hyporheic exchange for complex natural streambed topography, channel geometry and sediment structure. Ph. D. thesis, Northwestern University, USA; 2004.

[27] Cardenas MB, JL Wilson. Exchange across a sediment-water interface with ambient groundwater discharge. J Hydrol 2007;346(3-4):69-80.

[28] Cardenas MB, JL Wilson. Dunes, turbulent eddies, and interfacial exchange with permeable sediments. Water Resour Res 2007;43(8). doi:10.1029/2006WR005787.

[29] Cardenas MB. Surface water-groundwater interface geomorphology leads to scaling of residence times. Geophys Res Lett 2008;35(8). doi:10.1029/2008GL033753.

[30] Cardenas MB. The effect of river bend morphology on flow and timescales of surface water-groundwater exchange across pointbars. J Hydrol 2008;362(1-2):134-41.

[31] Cardenas MB, JL Wilson, VA Zlotnik. Impact of heterogeneity, bed forms, and stream curvature on subchannel hyporheic exchange. Water Resour Res 2004;40(8). doi:10.1029/2004wr003008

[32] Malard F, K Tockner, MJ Dole-Olivier, JV Ward. A landscape perspective of surface-subsurface hydrological exchanges in river corridors. Freshwater Biol 2002;47(4):621-40.

[33] Bencala K, V Kennedy, G Zellweger, A Jackman, R Avanzino. Interactions of solutes and streambed sediment 1. An experimental analysis of cation and anion transport in a mountain stream. Water 
Resour Res 1984;20(12):1797-803.

[34] Boano F, C Camporeale, R Revelli, L Ridolfi. Sinuosity-driven hyporheic exchange in meandering rivers. Geophys Res Lett 2006;33(18):L18406. doi:10.1029/2006GL027630.

[35] Revelli R, F Boano, C Camporeale, L Ridolfi. Intra-meander hyporheic flow in alluvial rivers. Water Resour Res 2008;44(12). doi:10.1029/2008WR007081.

[36] Marion A, M Zaramella. Diffusive behavior of bedform-induced hyporheic-exchange in rivers. J Environ Eng-ASCE 2005;131(9):1260-6.

[37] De Smedt F. Analytical solution and analysis of solute transport in rivers affected by diffusive transfer in the hyporheic zone. J Hydrol 2007;339(1-2):29-38.

[38] Richardson CP, AD Parr. Modified Fickian model for solute uptake by runoff. J Environ Eng-ASCE 1988;114(4):792-809.

[39] Qian Q, VR Voller, HG Stefan. A vertical dispersion model for solute exchange induced by underflow and periodic hyporheic flow in a stream gravel bed. Water Resour Res 2008;44(7). doi:10.1029/2007WR006366.

[40] O'Connor D. Models of sorptive toxic substances in freshwater systems. I. Basic equations. J Environ Eng-ASCE 1988;114(3):552-74.

[41] Zaramella M, AI Packman, A Marion. Application of the transient storage model to analyze advective hyporheic exchange with deep and shallow sediment beds. Water Resour Res 2003;39(7). doi:10.1029/2002WR001344.

[42] Elliott AH, NH Brooks. Transfer of nonsorbing solutes to a streambed with bed forms: Theory. Water Resour Res 1997;33(1):123-36.

[43] Bear J, A Verruijt. Modeling groundwater flow and pollution: with computer programs for sample cases. Kluwer Academic Publishers, Massachusetts, USA, 1987.

[44] Kinzelbach W. The random walk method in pollutant transport simulation, in E. Cusodio et al., Groundwater flow and and Quality Modelling, Reidel publishing Company, Boston, USA; 1988.

[45] Eylers H. Transport of adsorbing metal ions between stream water and sediment bed in a laboratory flume. Report No. KH-R-56, California Institute of Technology, USA; 1994.

[46] Eylers H, N Brooks, J Morgan. Transport of adsorbing metals from stream water to a stationary sand-bed in a laboratory flume. Mar Freshwater Res 1995;46(1):209-14.

[47] Packman AI, NH Brooks, JJ Morgan. A physicochemical model for colloid exchange between a stream and a sand streambed with bed forms. Water Resour Res 2000;36(8):2351-61.

[48] Packman AI, NH Brooks, JJ Morgan. Kaolinite exchange between a stream and streambed: Laboratory experiments and validation of a colloid transport model. Water Resour Res 2000;36(8):2363-72.

[49] Ren JH, AI Packman. Modeling of simultaneous exchange of colloids and sorbing contaminants between streams and streambeds. Environ Sci Technol 2004;38(10):2901-11.

[50] Ren JH, AI Packman. Coupled stream-subsurface exchange of colloidal hematite and dissolved zinc, copper, and phosphate. Environ Sci Technol 2005;39(17):6387-94.

[51] Cardenas MB, JL Wilson, R Haggerty. Residence time of bedform-driven hyporheic exchange. Adv Water Resour 2008;31(10):1382-6.

[52] Marion A, M Bellinello, I Guymer, A Packman. Effect of bed form geometry on the penetration of nonreactive solutes into a stream bed. Water Resour Res 2003;39(1). doi:10.1029/2001WR000264.

[53] Huettel M, W Ziebis, S Forster, GW Luther. Advective transport affecting metal and nutrient distributions and interfacial fluxes in permeable sediments. Geochim Cosmochim Acta 
1998;62(4):613-31.

[54] Chinese National Standard. Standard for soil test method. GB/T50123-1999, Beijing 1999 (in Chinese).

[55] Ren JH, AI Packman. Stream-subsurface exchange of zinc in the presence of silica and kaolinite colloids. Environ Sci Technol 2004;38(24):6571-81.

[56] Boano F, D Poggi, R Revelli, L Ridolfi. Gravity-driven water exchange between streams and hyporheic zones. Geophys Res Lett 2009;36(20):L20402. doi:10.1029/2009GL040147.

[57] Wilcox D. Turbulence modeling for CFD. DCW industries La Canada, California, 2006.

[58] Yoon JY, VC Patel. Numerical model of turbulent flow over sand dune. J Hydraul Eng-ASCE 1996;122(1):10-8.

[59] Alfrink BJ, LC Van Rijn. Two-equation turbulence model for flow in trenches. J Hydraul Eng-ASCE 1983;109(7):941-58.

[60] FLUENT. FLUENT 6.3 Documentation. Fluent Inc., Lebanon, NH, 2006.

[61] COMSOL. FEMLAB 3.3 Multiphysics Modeling - User's Guide. COMSOL Inc, Burlington, MA, USA, 2006.

[62] Bear J. Dynamics of fluids in porous media. Elsevier, New York, 1972.

[63] Neuman S. Universal scaling of hydraulic conductivities and dispersivities in geologic media. Water Resour Res 1990;26(8):1749-58.

[64] Kanel S, R Goswami, T Clement, M Barnett, D Zhao. Two dimensional transport characteristics of surface stabilized zero-valent iron nanoparticles in porous media. Environ Sci Technol 2008;42(3):896.

[65] Ullman W, R Aller. Diffusion coefficients in nearshore marine sediments. Limnol Oceanogr 1982;27(3):552-6.

[66] Iversen N, B Jorgensen. Diffusion coefficients of sulfate and methane in marine sediments: Influence of porosity. Geochimica Et Cosmochimica Acta 1993;57571-8.

[67] Alley W, R Healy, J LaBaugh, T Reilly. Flow and storage in groundwater systems. Science 2002;296(5575):1985-90.

[68] Gibbes B. Pore water exchange processes in offshore intertidal sandbanks: Ph. D. thesis, The University of Queensland, Australia; 2007. 


\section{Appendix: Determination of the solute front}

We assume that solute transport takes place in a flow tube (Fig. 5) with negligible transverse mixing across the tube and thus is governed by the $1 \mathrm{D}$ advection dispersion equation. Assuming a constant concentration boundary at the sediment-water interface, the analytical solution to the advective-dispersive equation (ADE) for a semi-infinite domain subject to a first-type condition at $x=0$ is:

$C(x, t)=\frac{C_{0}}{2}\left[\operatorname{erfc}\left(\frac{x-u t}{2 \sqrt{D t}}\right)+\exp \left(\frac{u x}{D}\right) \operatorname{erfc}\left(\frac{x+u t}{2 \sqrt{D t}}\right)\right]$

Based on this solution, we can see that when $t=x / u$, the concentration is approximately half $C_{0}$. Thus $C / C_{0}=0.5$ can be used to defined the solute front. 


\section{List of tables}

Table 1. Experimental conditions.

\begin{tabular}{ccccccccc}
\hline $\begin{array}{c}\text { Run } \\
\text { No. }\end{array}$ & $\begin{array}{c}\text { Streambed } \\
\text { depth } \\
(\mathrm{cm})\end{array}$ & $\begin{array}{c}\text { Water } \\
\text { depth } \\
(\mathrm{cm})\end{array}$ & $\begin{array}{c}\text { Stream } \\
\text { flow } \\
\text { velocity } \\
\left(\mathrm{cm} \mathrm{s}^{-1}\right)\end{array}$ & $\begin{array}{c}\text { Bedform } \\
\text { length }\end{array}$ & $\begin{array}{c}\text { Bedform } \\
\text { height } \\
(\mathrm{cm})\end{array}$ & $\begin{array}{c}\text { Stoss } \\
\text { length } \\
(\mathrm{cm})\end{array}$ & $\begin{array}{c}\text { Initial } \\
\text { concentration } \\
\left(\mathrm{g} \mathrm{l}^{-1}\right)\end{array}$ & $\mathrm{pH}$ \\
\hline 1 & 11.79 & 8.22 & 12.78 & 15.24 & 2.01 & 11.41 & 2.22 & 7.2 \\
2 & 11.45 & 8.56 & 12.56 & 15.27 & 1.80 & 10.15 & 2.10 & 7.1 \\
\hline \multicolumn{7}{l}{ water density $\rho=1000 \mathrm{~kg} \mathrm{~m}^{-3}$ and kinematic viscosity } & $v=10^{-6} \mathrm{~m}^{2} \mathrm{~s}^{-1}$. &
\end{tabular}


Table 2. Values of model parameters used in the numerical simulations of dispersion effects.

\begin{tabular}{|c|c|c|c|}
\hline Parameter & Value & Parameter & Value \\
\hline $\begin{array}{l}\text { Average velocity for } \\
\text { overlying water }(U)\end{array}$ & $0.2 \mathrm{~m} \mathrm{~s}^{-1}$ & Bulk porosity $(\theta)$ & 0.3 \\
\hline $\begin{array}{c}\text { Average water depth for } \\
\text { overlying water }(H)\end{array}$ & $0.5 \mathrm{~m}$ & Water density $(\rho)$ & $1000 \mathrm{~kg} \mathrm{~m}^{-3}$ \\
\hline Bedform length $(L)$ & $1 \mathrm{~m}$ & Water dynamic viscosity $(\mu)$ & $0.001 \mathrm{~Pa} \mathrm{~s}$ \\
\hline Bedform height $\left(H_{b}\right)$ & $5 \mathrm{~cm}$ & $\begin{array}{l}\text { Longitudinal dispersivity } \\
\qquad\left(\alpha_{L}\right)\end{array}$ & $0.001,0.01,0.1 \mathrm{~m}$ \\
\hline Stoss length $\left(L_{\mathrm{c}}\right)$ & $0.9 \mathrm{~m}$ & Transverse dispersivity $\left(\alpha_{T}\right)$ & $\begin{array}{c}0.0001,0.001,0.01 \\
\mathrm{~m}\end{array}$ \\
\hline Streambed depth $\left(d_{b}\right)$ & $1 \mathrm{~m}$ & $\begin{array}{l}\text { Effective diffusion coefficient } \\
\qquad\left(D_{e}\right)\end{array}$ & $10^{-9} \mathrm{~m}^{2} \mathrm{~s}^{-1}$ \\
\hline Intrinsic permeability $(\kappa)$ & $10^{-10} \mathrm{~m}^{2}$ & $\begin{array}{c}\text { Solute concentration in } \\
\text { overlying water }\left(C=C_{0}\right)\end{array}$ & $1 \mathrm{~kg} \mathrm{~m}^{-3}$ \\
\hline
\end{tabular}




\section{List of figures}

Fig. 1 (a) Schematic diagram of hyporheic zone in a river/stream system (after [67]).

(b) Pore water flows beneath a repeating bedform subject to unidirectional flow (after [68]). The pressure distribution (red line) along the sediment-water interface, induced by the current-bedform interaction, generates pore-water flow in the streambed. (c) Pore water flow in the streambed (after [27]). Arrows (with equal lengths) show the direction of pore water flow. The color scale represents the natural logarithm (for better visualization) of the pore water flow rate, with warm colors for high rates and cool for low rates. Streamlines are represented by black lines. The red streamline divides the streambed into two parts: the hyporheic zone and the ambient underflow zone.

(d) Solute transport processes in the streambed are shown by solute fronts (50\% of the input concentration, dashed line) at a given time and particle transport paths (line and arrow).

Fig. 2 Schematic diagram of the flume and other parts of the experimental apparatus.

Fig. 3 Schematic of simulation domain and boundaries. (a) For water flow (after [19]). $L, H, H_{b}$ and $d_{b}$ are bedform length, average water depth of overlying water, bedform height and average depth of streambed, respectively. (b) For solute transport. Uniform concentration is assumed in the overlying water. The total quantity of solute in the overlying water and the pore water did not vary with time in the flume experiment but the concentration in the overlying water did. For the purpose of simplicity, the solute concentration in the overlying 
water was kept constant at $1 \mathrm{~kg} \mathrm{~m}^{-3}$ over the simulation period in the additional simulations of dispersion effects.

Fig. 4 Comparison of experimental data and modeled solute concentration in the overlying water. Plot (a) is for Run 1 and (b) for Run 2.

Fig. 5 Two arrays of sampling ports for monitoring solute concentrations in the bed during the experiment for Run 1. Solid lines show the flow tube and travel times associated with port N1_P10 and port N2_P9, which were computed based on simulated flow velocities.

Fig. 6 Comparison between measured and predicted solute concentrations varying with depth for Run $1\left(C^{*}=C / C_{0}\right)$.

Fig. 7 Comparison between measured and predicted solute concentrations varying with time for Run 1.

Fig. 8 (a) Comparison of measured and simulated solute concentrations with those predicted by the advective transport model for Run 1 (based on $C_{s}(t)=C_{t}(t-$ $T$ ), where $T$ was calculated from the simulated flow field). Note that the data and simulation results for the overlying water concentration largely overlap. (b) Comparison between time of half peak concentration and solute slug travel time for Run 1.

Fig. 9 Solute concentration distribution (color) and particle travel path (solid line) in the streambed for adjusted $K$ and $\theta$ for the experiment Run 1. The dotted line indicates the location of the solute front (50\% of the peak concentration). The red line indicates the location of the advective solute front at $t=t_{c}$. 
Fig. 10 Comparison of solute concentration distribution (color) and particle transport path (solid line) for cases with different dispersivities. The simulated flow field is shown in the top panel. The green line shows the hydraulic divide separating the hyporheic zone and the ambient underflow zone. The dotted line indicates the location of the solute front $\left(50 \%\right.$ of $\left.C_{0}\right)$.

Fig. 11 Solute transport fluxes in the streambed for cases with different dispersivities at an early and late time: (a) magnitude (color) and direction (arrow) of advection fluxes ( $\theta C \mathbf{u}$ ), (b) magnitude (color) and direction (arrow) of dispersion flux $\left(-\theta D_{i j} \nabla C\right)$. Note that the natural logarithm of flux magnitude $\left(\mathrm{kg} \mathrm{m}^{-2} \mathrm{~s}^{-1}\right)$ is shown for better visualization. The hydraulic divide is indicated by the red line.

Fig. 12 Total vertical fluxes (integrated from $x_{1}=0$ to $L$ ) varying with depth (The approximate depth of the divide was $0.687 \mathrm{~m}$ between the hyporheic zone and ambient flow zone).

Fig. 13 Cumulative solute mass transferred from the overlying water to bed. The vertical dot-dashed line indicates the advection characteristic time scale $\left(t_{c}\right)$. 


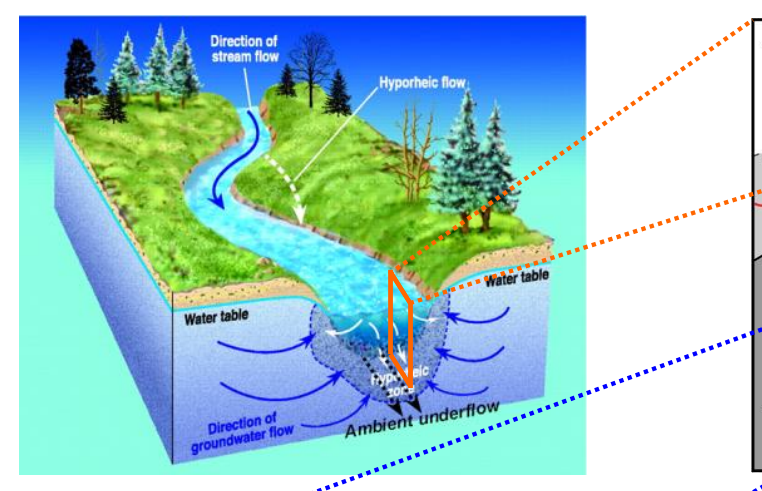

(a)

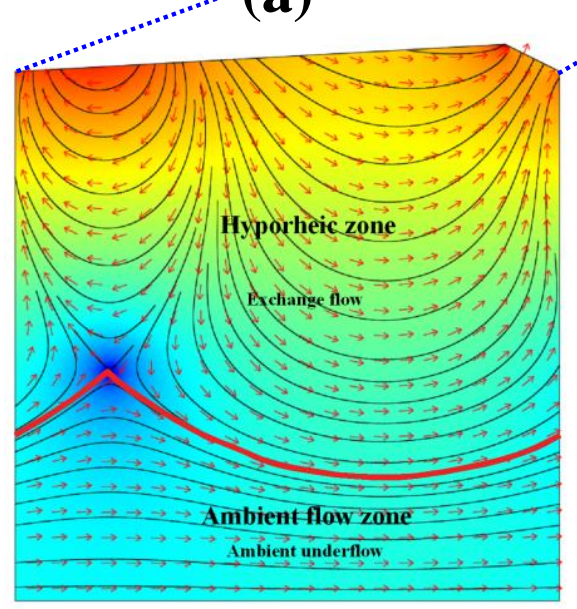

(c)

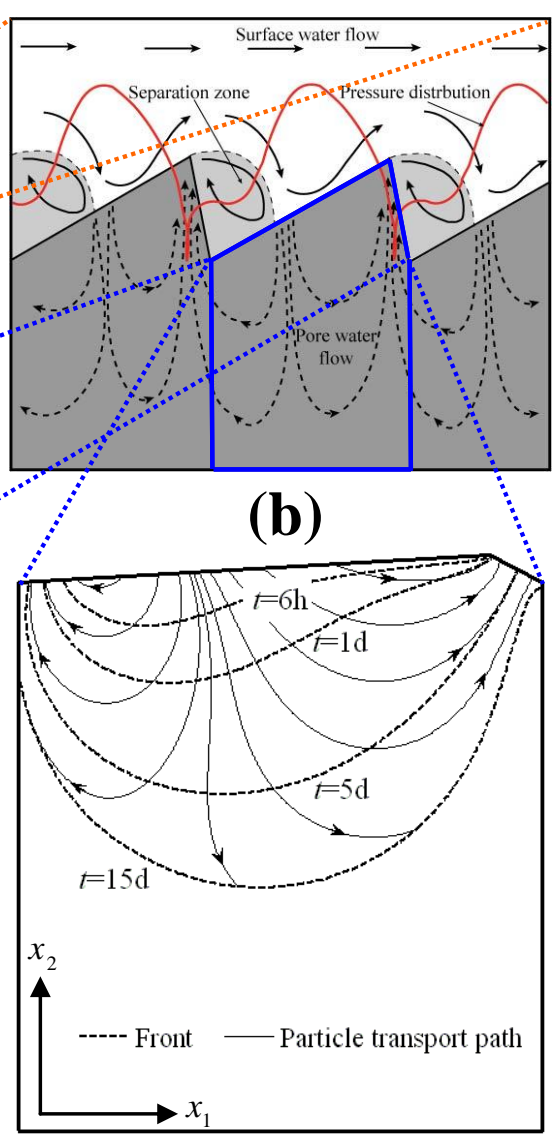

(d)

Fig. 1 


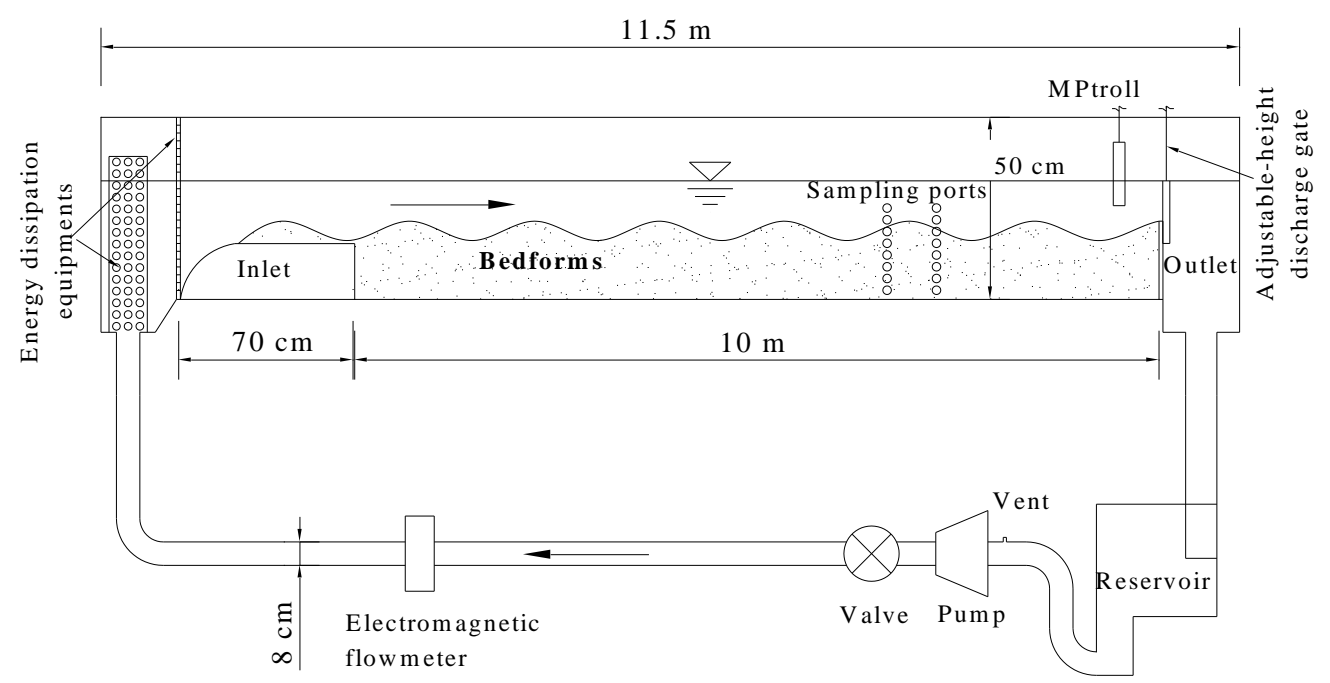

Fig. 2 


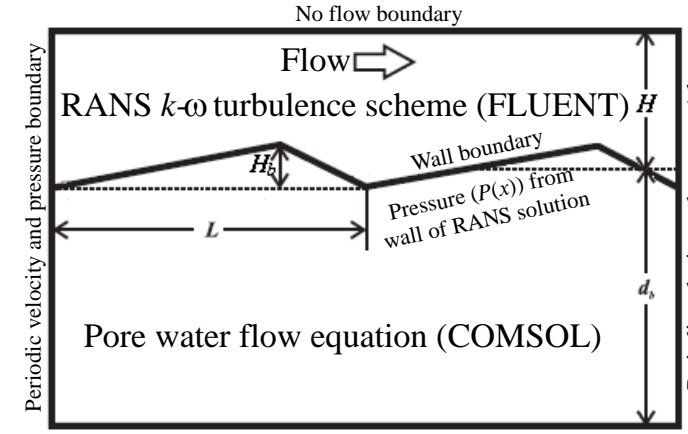

No flow boundary

(a)

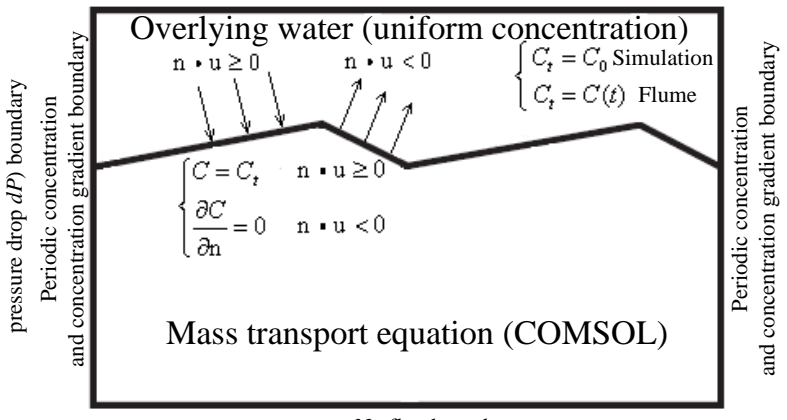

No flux boundary

(b)

Fig. 3 


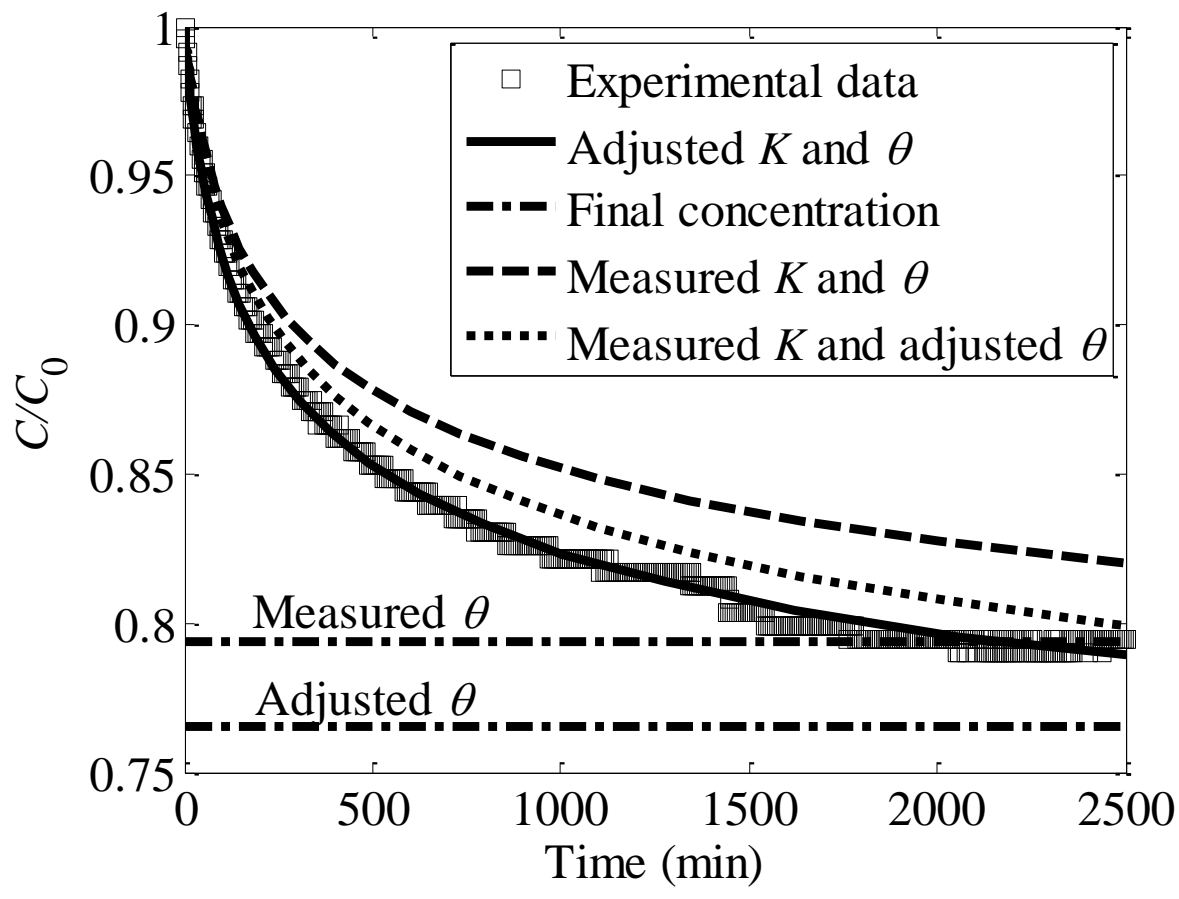

(a) for Run 1

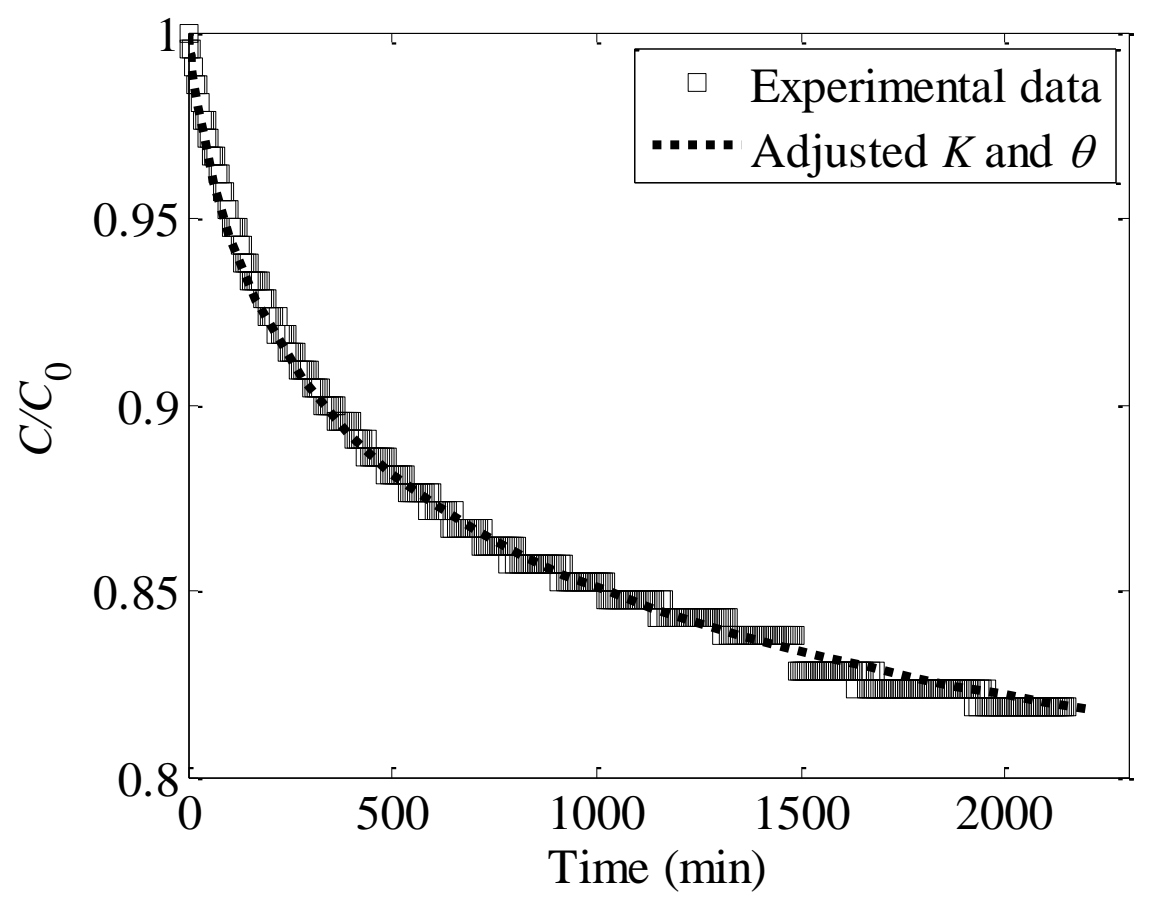

(b) for Run 2

Fig. 4 


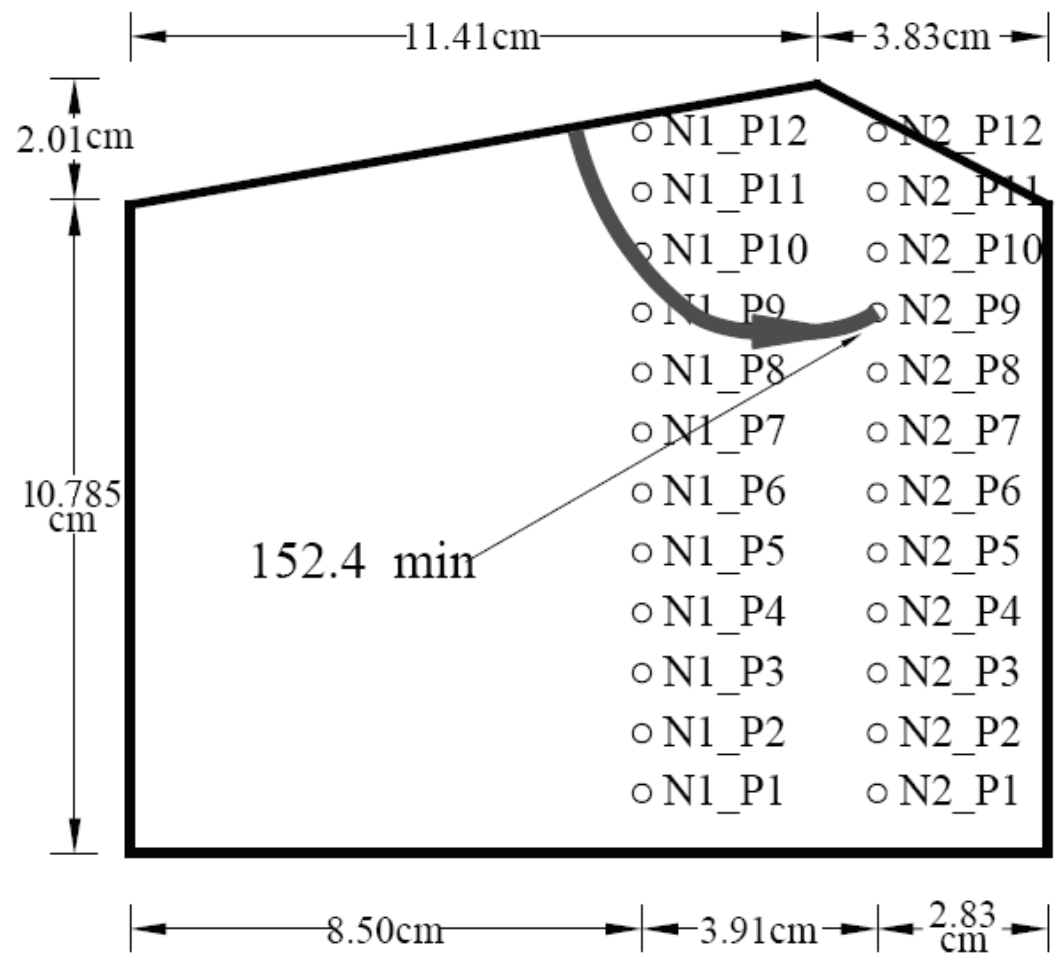

Fig. 5 

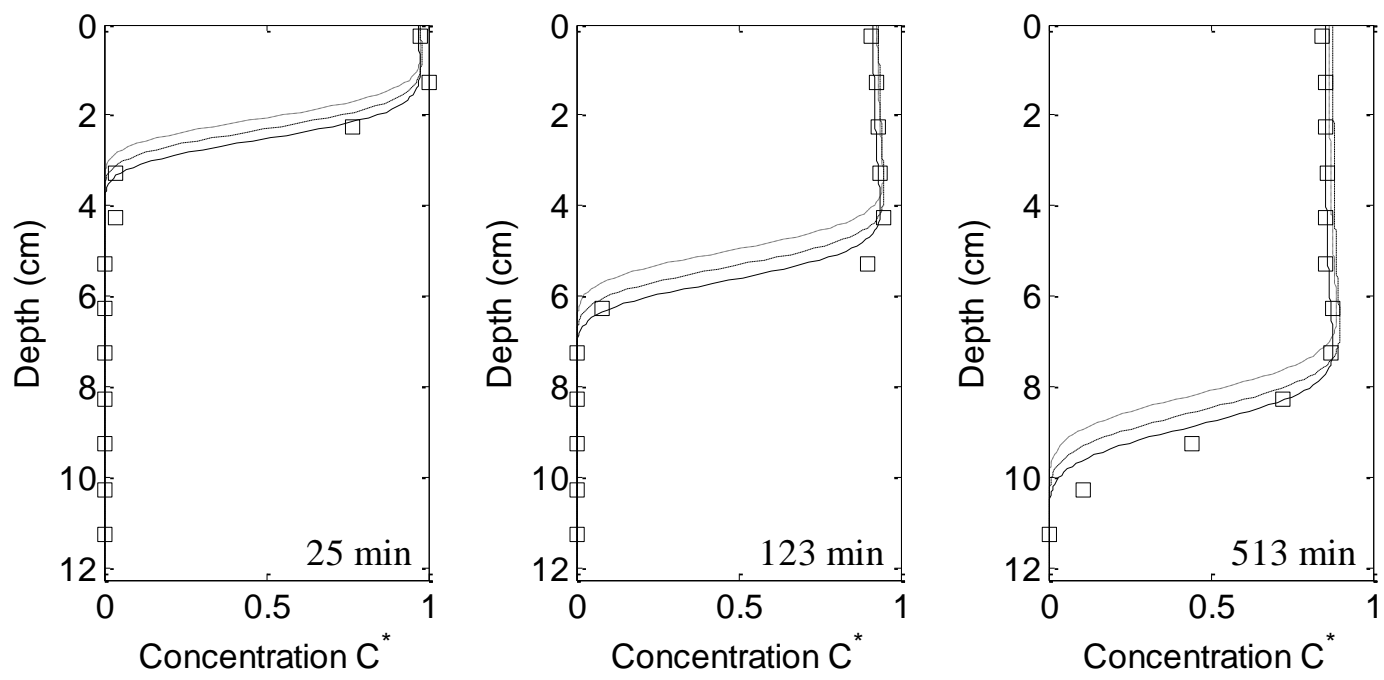

(a) array N1
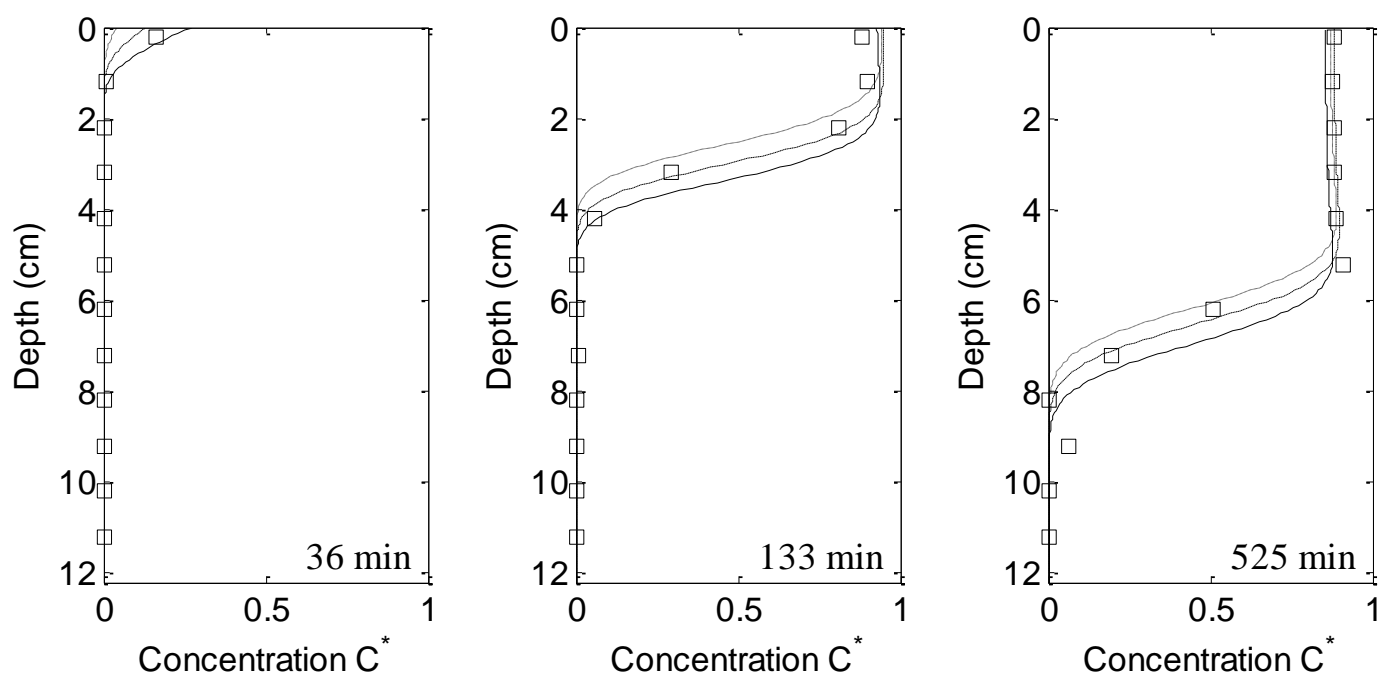

(b) array N2

$\square$ Experimental data - Adjusted $K$ and $\theta \quad--$ Measured $K$ and $\theta \quad \cdots \cdot$ Measured $K$ and adjusted $\theta$

Fig. 6 

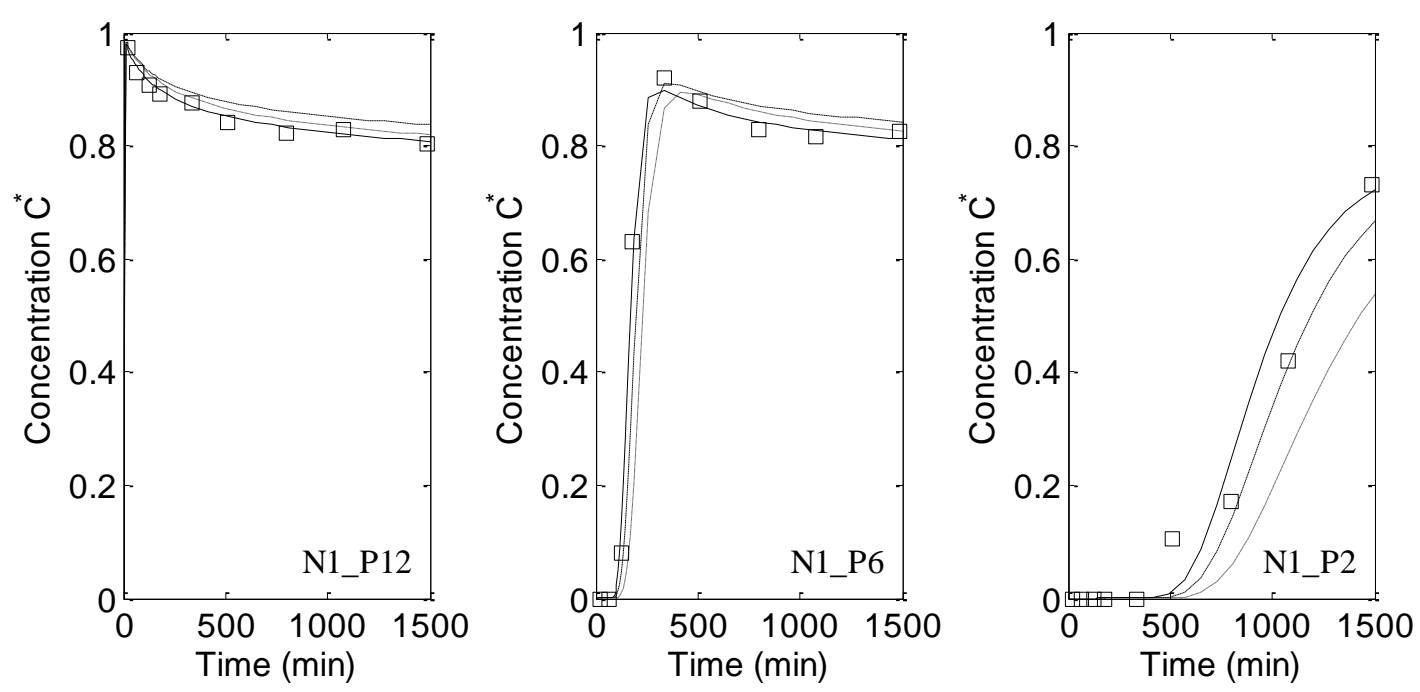

(a) array $\mathrm{N} 1$
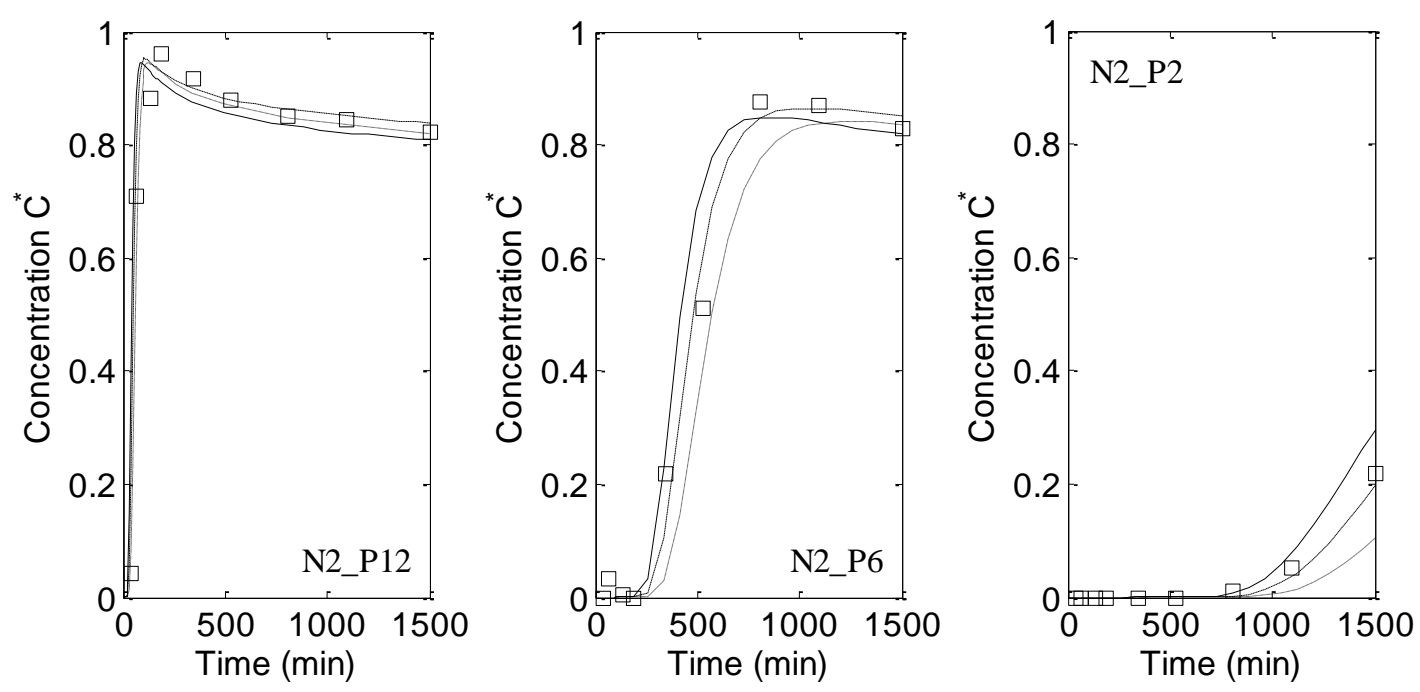

(b) array $\mathrm{N} 2$

$\square$ Experimental data - Adjusted $K$ and $\theta \quad---$ Measured $K$ and $\theta \quad \cdots \cdot$ Measured $K$ and adjusted $\theta$

Fig. 7 

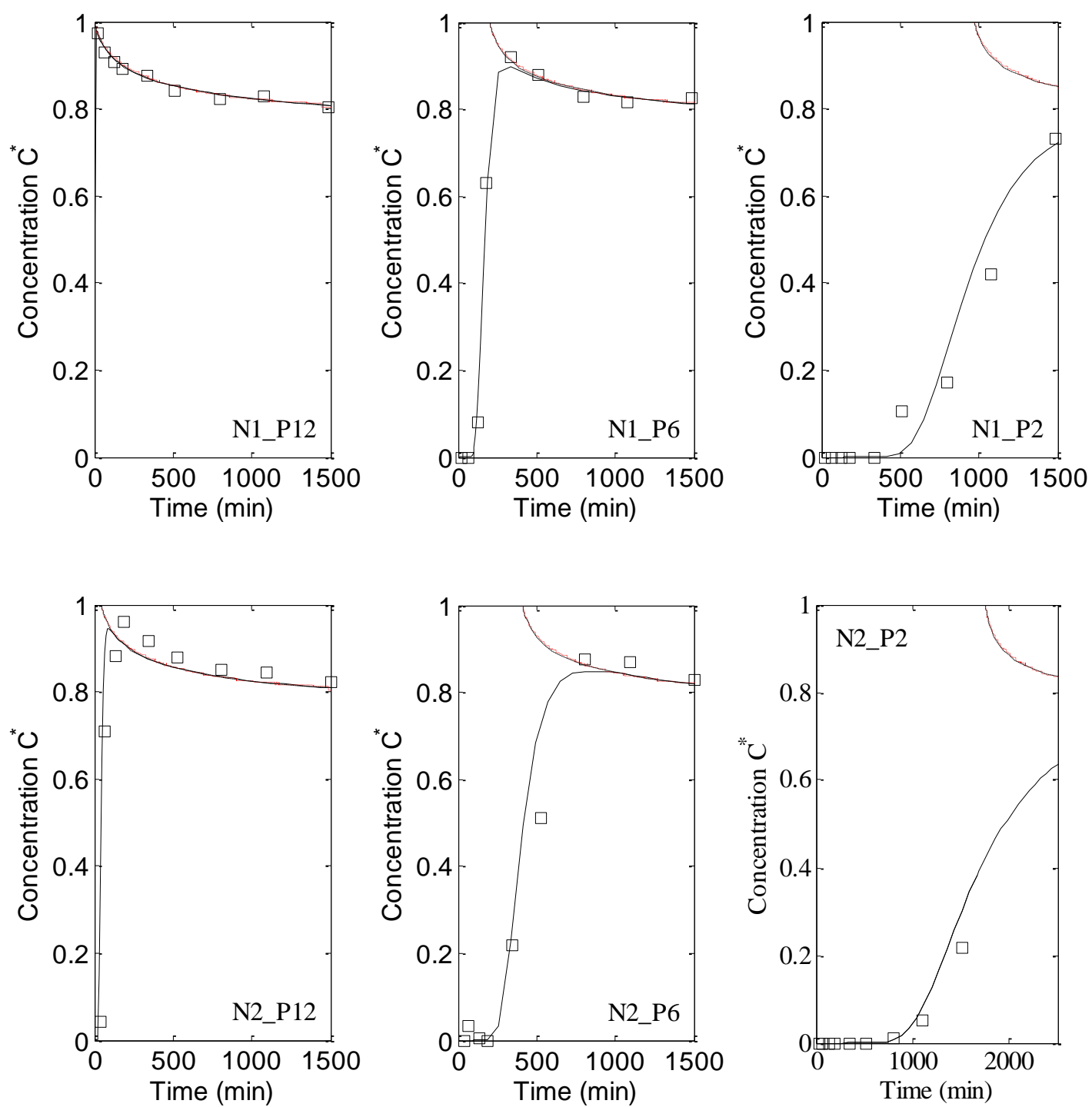

Experimental data for pore water $\longrightarrow$ Simulation for pore water

..... Experimental data for overlying water - - - Simulation for overlying water

Fig. 8(a) 


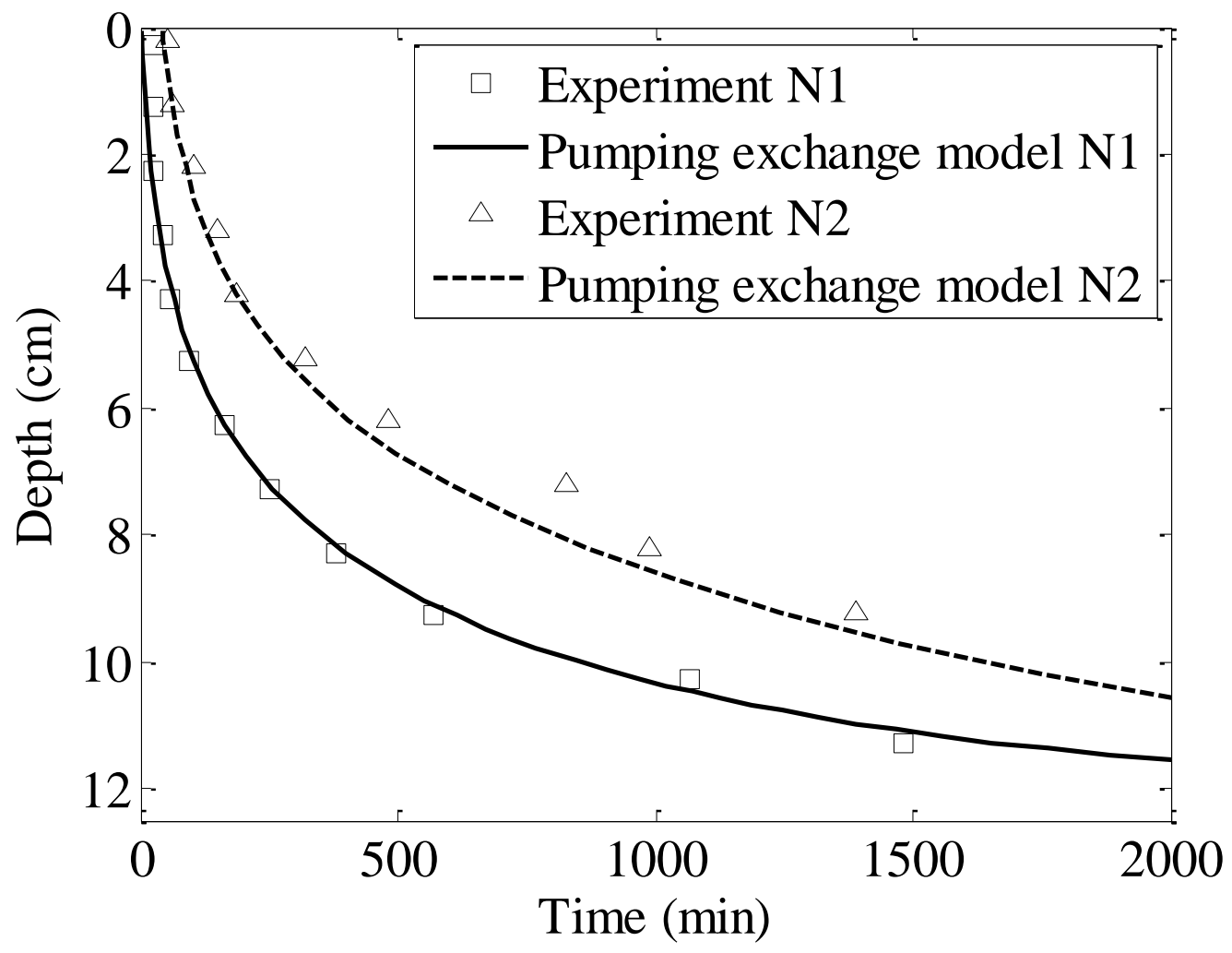

Fig. 8(b) 


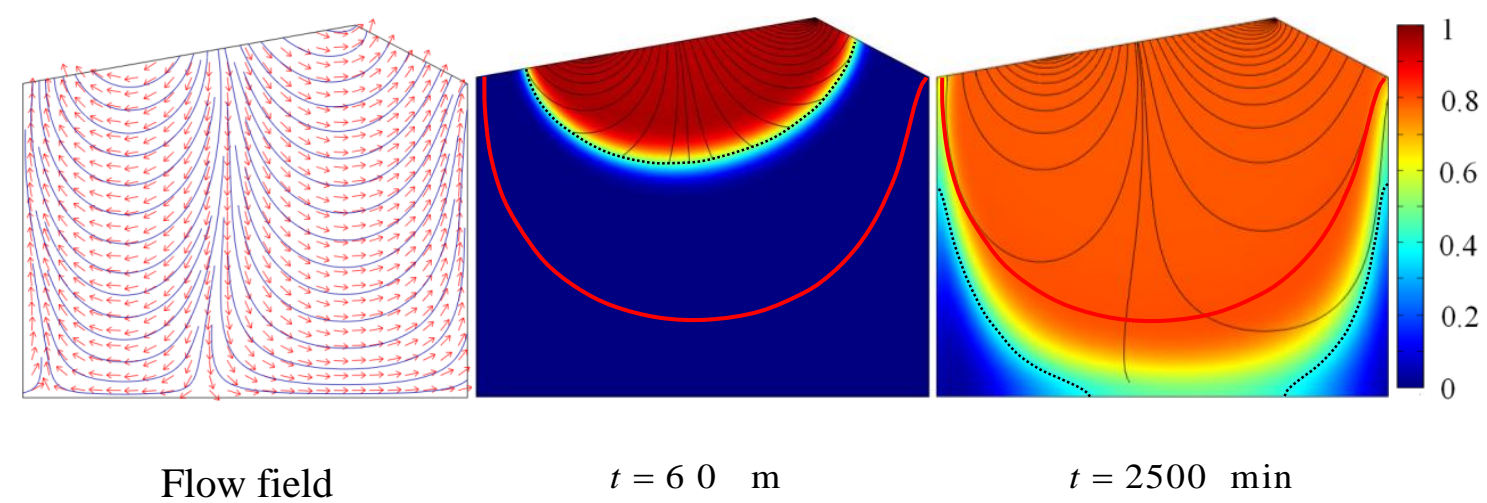

Fig. 9 


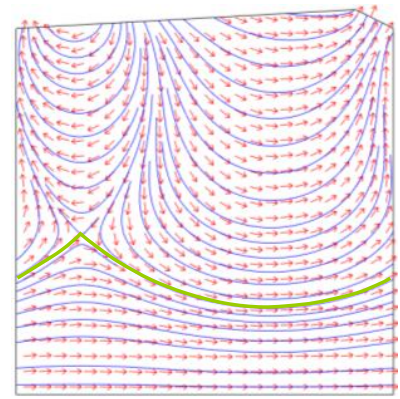

Flow field

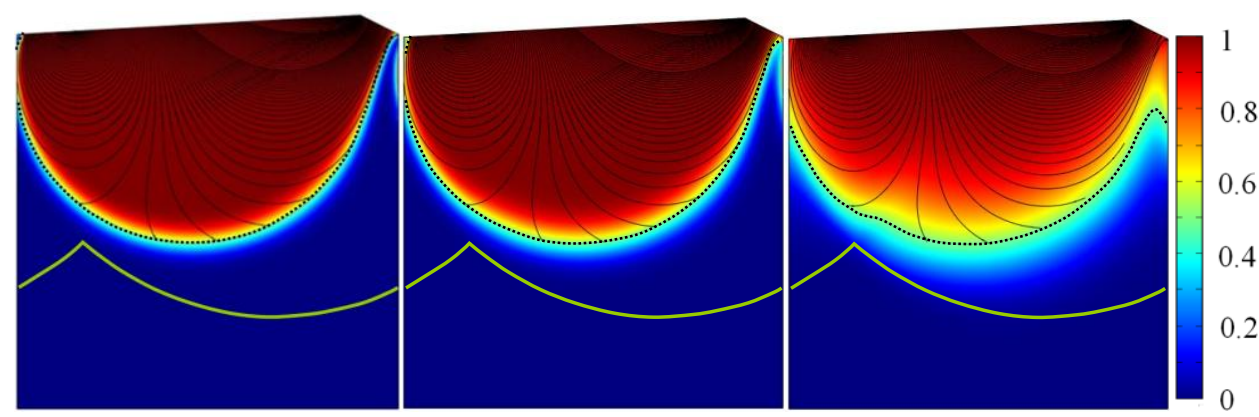

$t=15 \mathrm{~d}, \alpha=0.001 \mathrm{~m} \quad t=15 \mathrm{~d}, \alpha=0.01 \mathrm{~m} \quad t=15 \mathrm{~d} \alpha=\quad 0$

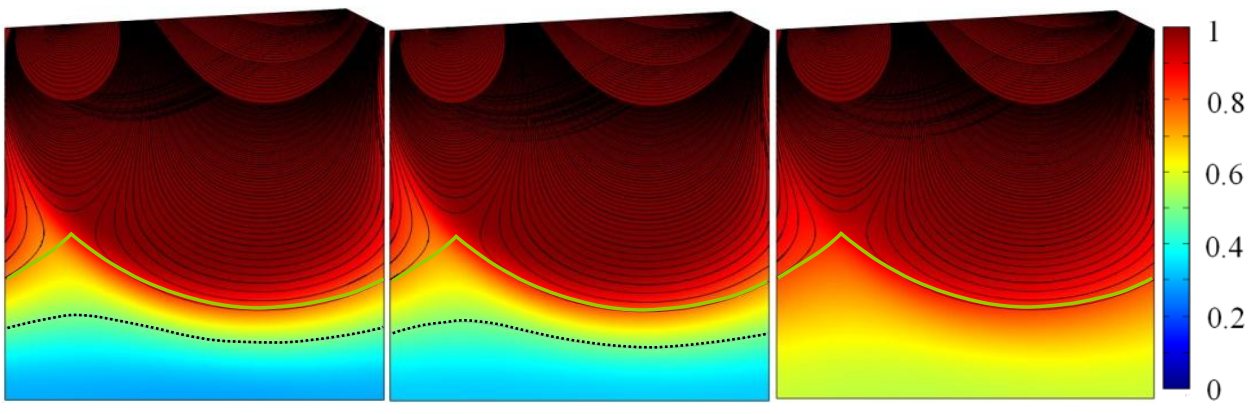

$t=1 \mathrm{y}, \alpha=0.001 \mathrm{~m} \quad t=1 \mathrm{y}, \quad \alpha=0.01 \mathrm{~m} \quad t=1 \quad \mathrm{y}, \alpha=\quad 0$.

Fig. 10 


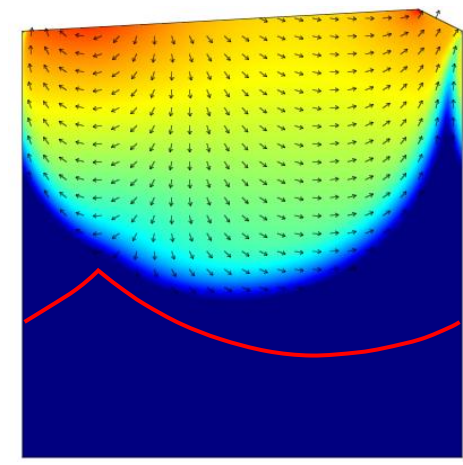

$t=15 \mathrm{~d}, \quad \alpha=0.001 \mathrm{~m}$

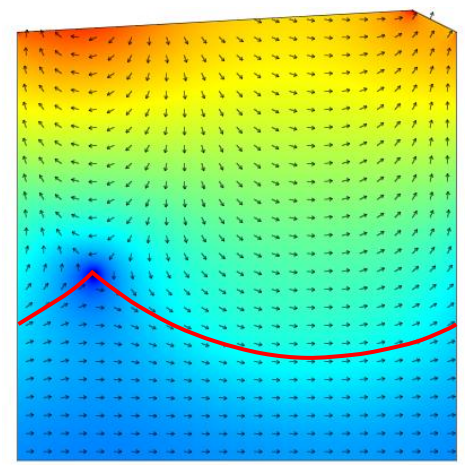

$t=1 \mathrm{y}, \quad \alpha=0.001 \mathrm{~m}$

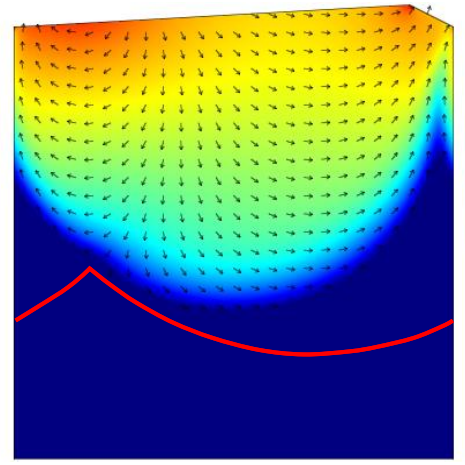

$t=15 \mathrm{~d} \alpha=0$.

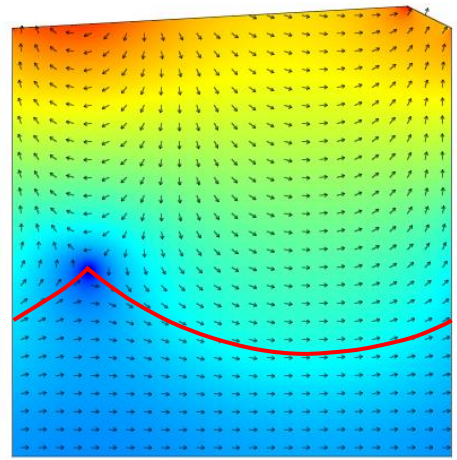

$t=1 \quad \mathrm{y}, \alpha=$

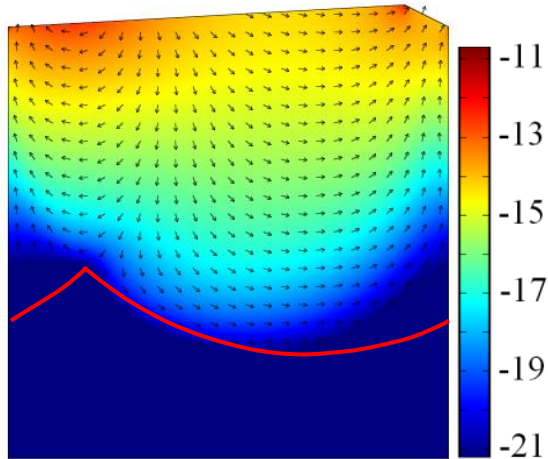

$t=15 \mathrm{~d}, \quad \alpha=0.1 \mathrm{~m}$

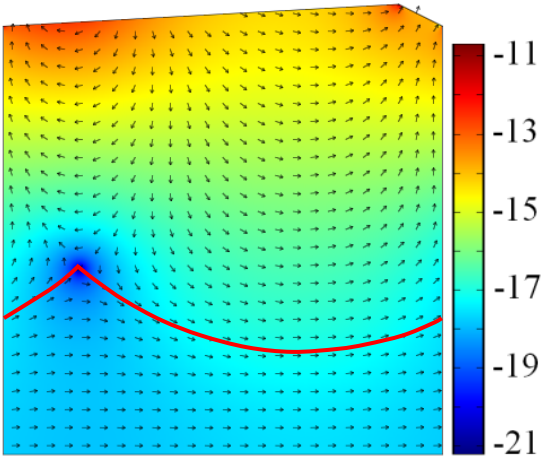

$t=1 \quad \mathrm{y}, \alpha=$
0 .

Fig. 11(a) 

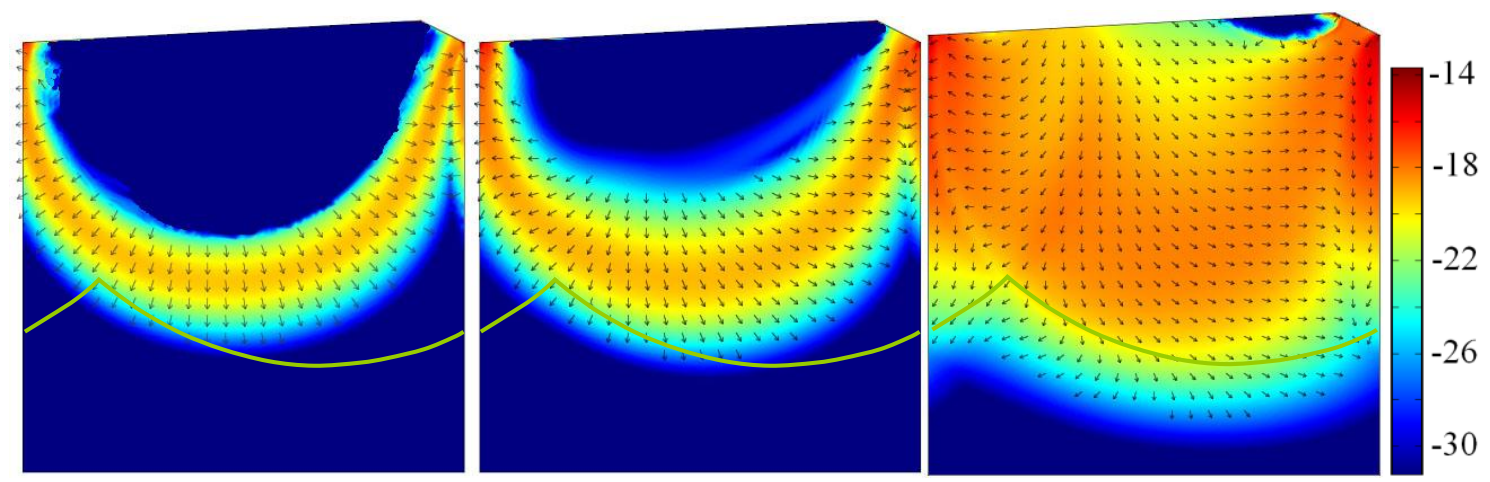

$t=15 \mathrm{~d}, \alpha=0.001 \mathrm{~m}$

$$
t=15 \mathrm{~d} \alpha=0 .
$$

$t=15 \mathrm{~d} \alpha=0$
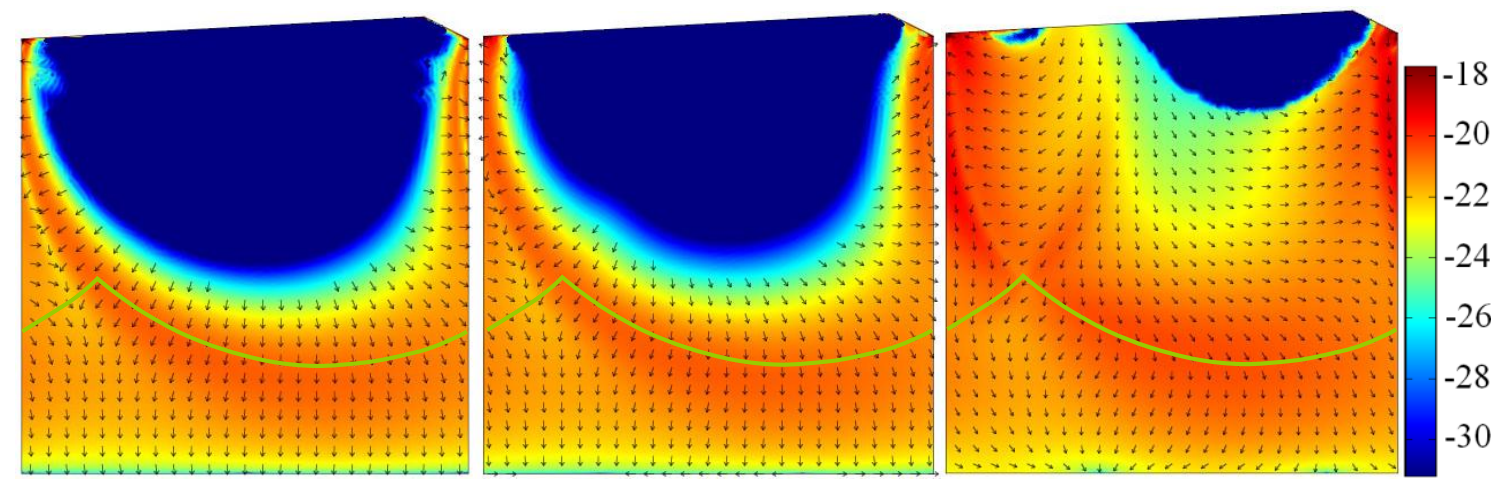

$t=1 \mathrm{y}, \quad \alpha=0.001 \mathrm{~m}$

$t=1 \quad \mathrm{y}, \alpha=0.1$

$t=1 \quad \mathrm{y}, \alpha=$

0 .

Fig. 11(b) 

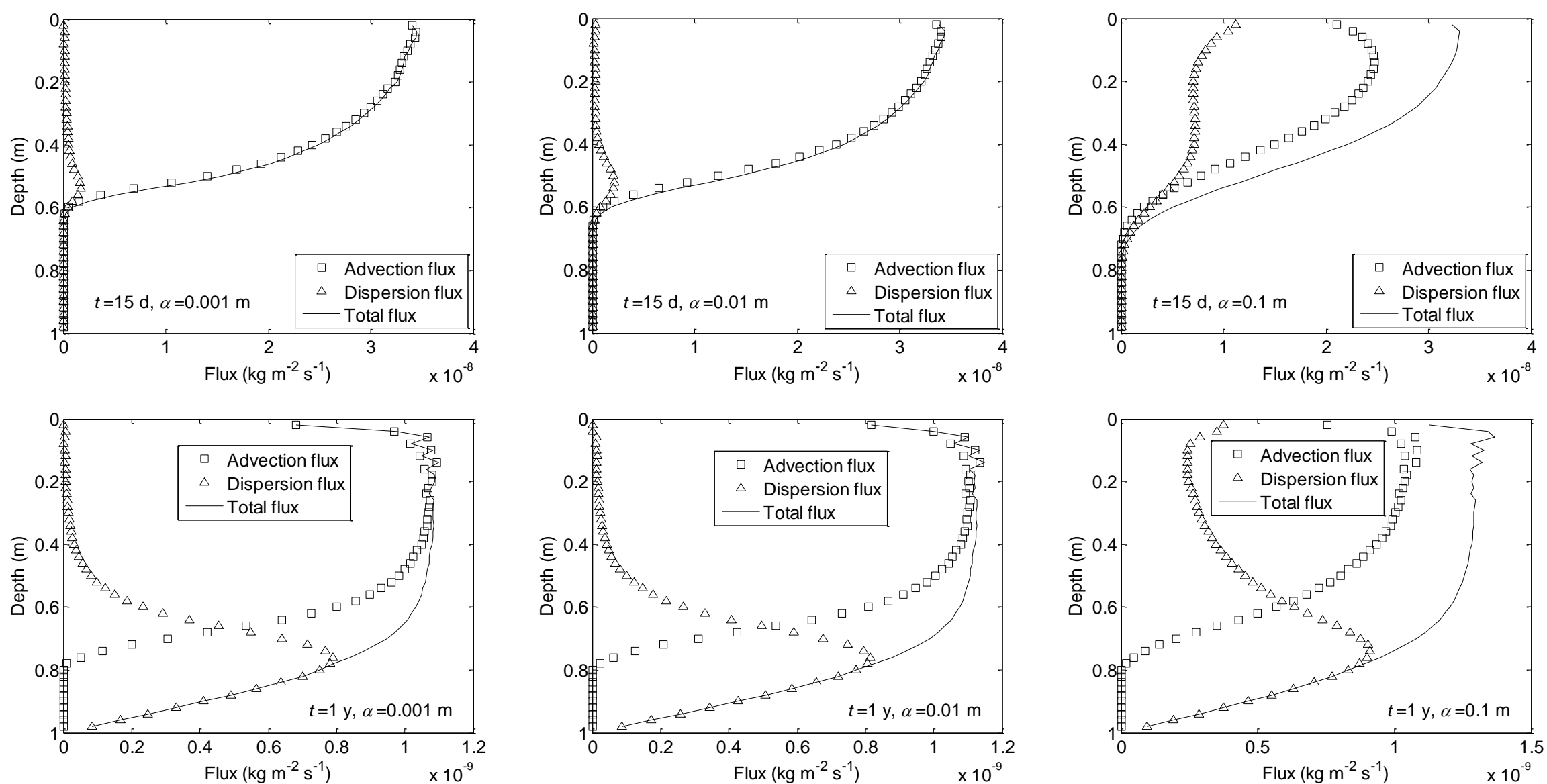

Fig. 12 


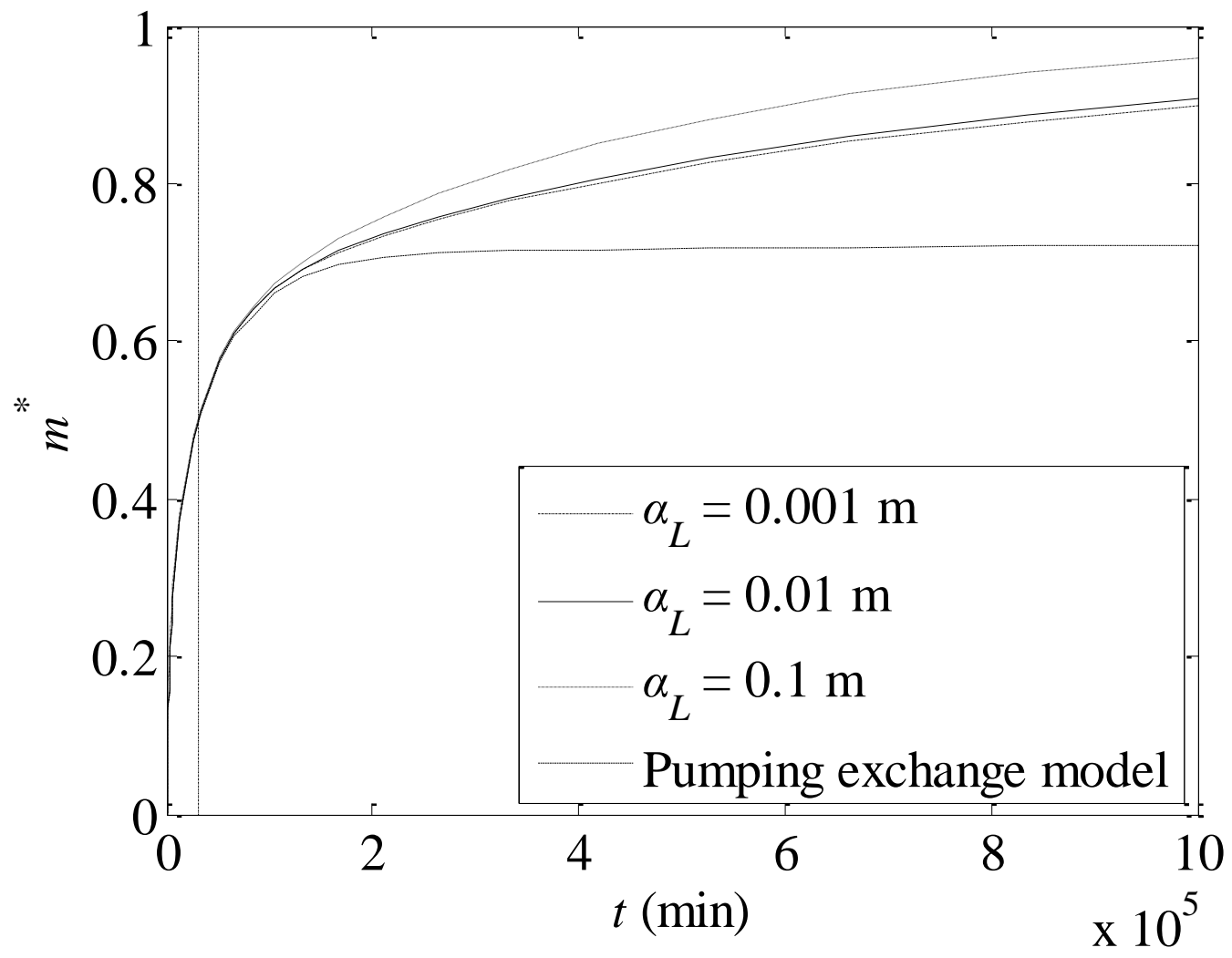

Fig. 13 